Annu. Rev. Earth Planet. Sci. 2013, Vol 41:469-95

\title{
The Formation and Dynamics of Super-Earth Planets
}

\author{
NADER HAGHIGHIPOUR \\ Institute for Astronomy and NASA Astrobiology Institute, University of \\ Hawaii, Honolulu, HI 96822, USA, Email:nader@ifa.hawaii.edu
}

Key Words planetary system: formation, planetary system: dynamics

\begin{abstract}
Super-Earths, objects slightly larger than Earth and slightly smaller than Uranus, have found a special place in exoplanetary science. As a new class of planetary bodies, these objects have challenged models of planet formation at both ends of the spectrum and have triggered a great deal of research on the composition and interior dynamics of rocky planets in connection to their masses and radii. Being relatively easier to detect than an Earth-sized planet at $1 \mathrm{AU}$ around a G star, super-Earths have become the focus of worldwide observational campaigns to search for habitable planets. With a range of masses that allows these objects to retain moderate atmospheres and perhaps even plate tectonics, super-Earths may be habitable if they maintain long-term orbits in the habitable zones of their host stars. Given that in the past two years a few such potentially habitable super-Earths have in fact been discovered, it is necessary to develop a deep understanding of the formation and dynamical evolution of these objects. This article reviews the current state of research on the formation of super-Earths and discusses different models of their formation and dynamical evolution.
\end{abstract}

\section{CONTENTS}

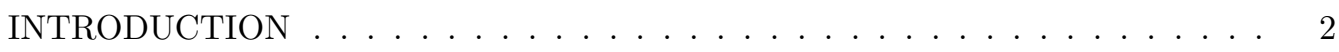

MODELS OF PLANET FORMATION ................... 3

FORMATION OF SUPER-EARTHS . . . . . . . . . . . . . . . . . 8

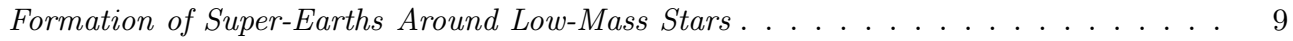

The Core-Accretion Model . . . . . . . . . . . . . . . . . . . . . 10

Effect of stellar evolution . . . . . . . . . . . . . . . . . . . . . . . . . . . . . . . . . . . . . . . . . . . . . . . . . .

Effect of planet migration . . . . . . . . . . . . . . . . . . . . . . . . . . . . . . . . . . . . . . . . . . .

The Disk-Instability Model . . . . . . . . . . . . . . . . . . . . . . 13

CONCLUDING REMARKS ........................ 14

ACKNOWLEDGMENT . . . . . . . . . . . . . . . . . . 14

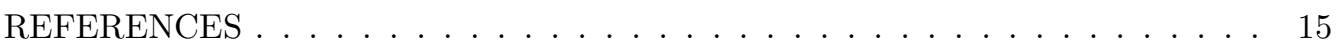




\section{INTRODUCTION}

The discovery of planets around other stars has undoubtedly revolutionized our understanding of the formation and dynamical evolution of planetary systems. The diverse and surprising characteristics of these objects, both in orbital configuration and physical properties, have confronted astronomers with many new challenges and have reinvigorated the fields of planet formation and dynamics.

One surprising characteristic of the currently known extrasolar planets is the range of their masses. Unlike in the Solar System, where planets belong to two distinct categories of terrestrial (with masses equal to that of Earth or slightly smaller) and giant [ 14 Earth masses $\left(M_{\oplus}\right)$ and larger], many extrasolar planets have masses in an intermediate range, from slightly larger than Earth to $10 M_{\oplus}$. Dubbed super-Earths, these objects present a new class of planetary bodies with physical and dynamical properties that for the past few years have been the focus of research among many planetary scientists.

The first super-Earth around a main sequence star was discovered by Rivera et al. (2005) using the radial velocity technique. [Note that in 1992, Wolszczan \& Frail (1992) discovered at least two terrestrial-class planets around the pulsar PSR $1257+12$.] Thanks to ground-based observational projects such as the HARPS Search for Southern Extrasolar Planet 1 , the California Planet Survey (CPS)2, the Lick-Carnegie Exoplanet Survey (LCE), M2K (Clubb et al. 2009), and the MEarth Project (Nutzman \& Charbonneau 2008; Irwin et al. 2009a,b)3, and the ongoing success of the CoRoT 4 and Kepler 5 space telescopes, to date, the number of these objects has exceeded 90. Tables 1 and 2 show the masses and orbital elements of the currently known super-Earths. As shown, the vast majority of these objects have orbital periods smaller than 50 days. A survey of the parent stars of these bodies indicates that more than half of these stars are hosts to multiple planets. This implies that super-Earths may be more likely to form in short-period orbits and in systems with multiple bodies - two characteristics that play important roles in developing models of their formation and dynamical evolution.

Among the currently known super-Earths, a few have gained special attention. CoRoT-7 b, the seventh planet discovered by the CoRoT space telescope (Léger et al. 2009; Queloz et al. 2009; Hatzes et al. 2010, 2011), and GJ 1214 b, the first super-Earth discovered by transit photometry around an M star (Charbonneau et al. 2009), are the first super-Earths for which the values of mass and radius have been measured [CoRoT-7 b: $2.38 M_{\oplus}, 1.65$ Earth radii $\left(R_{\oplus}\right)$; GJ 1214 b: $5.69 M_{\oplus}, 2.7 R_{\oplus}$ ]. This major achievement has enabled theoreticians to develop models for the evolution of super-Earths interiors (e.g., Valencia et al. 2006, 2007a,b,c, 2009, 2010; ONeill \& Lenardic 2007; Sotin \& Schubert 2009; Tackley \& van Heck 2009) and their possible atmospheric properties (e.g., Miller-Ricci et al. 2009; Seager \& Deming 2009; Bean et al. 2010; Miller-Ricci \& Fortney 2010; Rogers \& Seager 2010a,b; Bean et al. 2011; Désert et al. 2011; Heng \& Vogt 2011; Berta et al. 2012; Menou 2012; Fraine et al. 2013). The three superEarth-class bodies GL 581 d (Mayor et al. 2009, Forveille et al. 2011), GL 581

\footnotetext{
${ }^{1}$ http://www.eso.org/sci/facilities/lasilla/instruments/harps/

${ }^{2}$ http://www.exoplanets.org/cps.html

${ }^{3}$ http://www.cfa.harvard.edu/MEarth/Welcome.html

${ }^{4}$ http://smsc.cnes.fr/COROT/index.htm

${ }^{5}$ http://kepler.nasa.gov/
} 
g (Vogt et al. 2010, 2012), and GJ 667C c (Anglada-Escudé et al. 2011) have also made headlines. These planets are the first terrestrial-class objects that have been discovered in their respective habitable zones.

For the past few years, the formation and characteristics of super-Earths have been the subject of extensive research.This is primarily because being slightly larger than a typical terrestrial planet, these objects have the capability of developing moderate atmospheres and may have dynamic interiors with plate tectonics - two conditions that would render a super-Earth potentially habitable if its orbit were in the habitable zone of its host star (see Haghighipour 2011 for a complete review). Also, unlike Earth-sized planets, super-Earths are relatively easy to

detect. Current observations of super-Earths have indicated that these objects seem to be more common around cool and low-mass stars (see, e.g., Dressing \& Charbonneau 2013, Swift et al. 2013), where the habitable zone is in closer orbit. Two prime examples of such systems are GL 581, an M3V star with one or two potentially habitable super-Earths (Mayor et al. 2009; Vogt et al. 2010, 2012; Forveille et al. 2011), and the M1.5 star GJ $667 \mathrm{C}$, with a $4.5 M_{\oplus}$ planet in its habitable zone (Anglada-Escudé et al. 2011).

Given the success of observational techniques in detecting potentially habitable super-Earths, and that during the past two years the number of these objects increased twofold, it would be natural to expect that many more habitable superEarths will be detected in the near future. It is, therefore, imperative to develop a thorough understanding of the formation and dynamical evolution of these bodies, particularly in connection with their habitability. This article presents a review of the current state of research on this topic.

Since there are no super-Earths in the Solar System, it is important to know whether the formation of these objects requires developing new models of planet formation or whether one can use the models of the formation of planets in the Solar System to explain the formation of super- Earths. In the latter case, these models will require major revisions. For instance, one characteristic of super-Earths that presents a challenge to the theories of planet formation is their close-in orbits. While some models suggest that super-Earths were formed at large distances and migrated to their present locations, other models present the possibility of their in-place formation. Fortunately, the physical characteristics of super-Earths, namely their densities, when considered within the context of different planet formation scenarios, present a potential pathway for differentiating between these models. In that respect, the study of super-Earths plays an important role in identifying the most viable planet formation mechanism. The rest of this article presents a review of the current state of research on this topic.

I begin in Section 2 by briefly reviewing the models of planet formation in the Solar System. In Section 3, I discuss in detail the application of these models to the formation of super-Earths, and I conclude in Section 4.

\section{MODELS OF PLANET FORMATION}

Explaining the formation of planets is one of the most outstanding problems in planetary astronomy. Despite centuries of efforts to explain the formation of the planets of the Solar System, this problem is still unresolved, and planet formation is still an open question. The discovery of extrasolar planets has added even more to these complexities. As explained in Section 1, many of these objects 
have physical and orbital properties that are unlike those of the planets in the Solar System and are not well explained by the current models of Solar System formation and dynamics.

Although the diversity of extrasolar planets has been a continuous challenge to the models of planet formation, a common practice in explaining the formation of these objects has been to modify, revise, and/or complement the models of planet formation in the Solar System in such a way that they would be applicable to other planetary bodies. This suggests that to understand the formation of extrasolar planets (such as super-Earths), it is necessary to develop a deep understanding of the models of giant and terrestrial planet formation in the Solar System. This section is devoted to this task. I begin by explaining the growth of dust particles to larger bodies, then discuss different phases of planet growth until a full giant or terrestrial planet is formed.

It is widely accepted that planet formation begins in a circumstellar disk of gas and dust known as a nebula by the growth of dust particles to larger objects. This process, highly dependent on the mass and dynamical properties of the nebula, proceeds in four stages:

- coagulation of dust particles through gentle hitting and sticking, which results in the formation of centimeter- and decimeter-sized objects;

- growth of centimeter- and decimeter-sized bodies to kilometer-sized planetesimals;

- collision and accretion of planetesimals to planetary embryos (moon- toMarssized objects) in the inner part of the Solar System and to the cores of giant planets in the outer parts; and

- the accretion of gas and formation of giant planets followed by the collisional growth of planetary embryos to terrestrial-class bodies.

The first stage of this process is well understood. Dust grains at this stage undergo different types of random and systematic motions (Weidenschilling 1977) and frequently collide with one another. Particles smaller than $100 \mu \mathrm{m}$ are mainly subject to Brownian motion and collide with relative velocities smaller than 1 mm s1. Larger objects, although slightly faster, are still strongly coupled to the gas, and their dynamics is governed by the gravitational attraction of the central star, nongravitational forces such as radiation pressure, and their interaction with the nebula through gas drag. Gas molecules, however, are subject to pressure gradient (which is necessary for maintaining the gas at hydrostatic equilibrium), and as a result, their velocities are slightly smaller than Keplerian. The slight velocity differences between dust particles and gas molecules cause dust grains to drift inward and approach one another with small relative velocities (Safronov 1969; Weidenschilling 1980; Nakagawa et al. 1981, 1986; Supulver \& Lin 2000; Dullemond \& Dominik 2005). Turbulence also causes dust grains to collide and is more effective among same-sized particles. As the collisions of dust particles are gentle, van der Waals forces act between their surfaces and stick the dust particles to one another. As shown by laboratory experiments and computational simulations, such gentle collisions result in the fractal growth of dust grains to larger aggregates (Figure 1) (Smoluchowski 1916; Dominik \& Tielens 1997; Blum et al. 1998; Wurm \& Blum 1998; Blum \& Wurm 2000; Krause \& Blum 2004; Blum 2006, 2010; Wada et al. 2007).

While the process of the growth of micrometer-sized dust grains to millimeter- 
and centimeter-sized objects is well understood, the growth of the latter bodies to larger sizes (i.e., kilometer size) is still a big mystery. Simulations have shown that as dust particles grow, their coupling to the gas weakens (i.e., their velocities relative to the gas molecules increase), and they show more of their independent dynamics (Weidenschilling 1977). At this stage, differential vertical settling (Safronov 1969), radial drift (Whipple 1972), and turbulence (Völk et al. 1980, Mizuno et al. 1988, Ormel \& Cuzzi 2007) play important roles in driving particles relative velocities. The latter causes objects to approach each other rapidly and increases their impact velocities. Results of laboratory experiments and computational simulations have shown that as objects grow to centimeters in size, their sticking efficiency drops dramatically (Blum \& Münch 1993), and their relative velocities become so large that their collisions may result in bouncing (bouncing barrier) and/or erosion and fragmentation (fragmentation barrier) (Blum \& Wurm 2008, Güttler et al. 2009, Zsom et al. 2010, Beitz et al. 2011).

The above-mentioned bouncing and fragmentation barriers are not the only obstacles in the formation of planetesimals. The sub-Keplerian rotational velocities of gas molecules result in the transfer of angular momentum from solid bodies to the gas and the subsequent drift of these objects toward the central star. The rate of this radial drift is approximately proportional to the size of an object, implying that as an object grows, it approaches the central star in a shorter time. Numerical simulations have indicated that meter-sized bodies have the fastest radial drifts. Combined with turbulence and differential settling, this radial drift increases the relative velocities of solid objects and causes many of them to collide with one another at large speeds. Given that large objects are more prone to collisional destruction (the sticking properties of solid bodies weaken as they grow), it is expected that many of these impacts result in the breakage of the colliding bodies. This process, known as the meter-size barrier, implies that even if the centimeter-size bouncing barrier is overcome, the impact velocities of solid objects become so large that their collisions result in their breaking into small fragments, which subsequently halts their growth to larger sizes. These fragments, even if reaccumulated, will go through the same above-mentioned process and ultimately drift into the central star, leaving the nebula devoid of the solid material necessary for the formation of planetesimals.

Interestingly, despite all these difficulties, planets do exist and so do many kilometer-sized bodies, such as the asteroids and Kuiper belt objects. This implies that during the early stages of planet formation, Nature succeeded in finding a way to overcome the centimeter-sized and meter-sized barriers. It may be that kilometer-sized planetesimals did not form as a result of the mere collisional growth of dust grains; other mechanisms may have also contributed.

A planet-forming nebula is a dynamic environment whose properties and structure vary with time. These variations, in particular in a gaseous disk, may manifest themselves as different structures in the nebula. For instance, regions may appear where the pressure of the gas is locally enhanced. The appearance of such structures will immediately affect the motions of particles in their surroundings. As opposed to a nebula with a monotonic radial pressure profile where gas drag and pressure gradient cause inward migration of solids, in the vicinity of pressure-enhanced regions, the velocity differences between solid objects and gas molecules cause solid particles to undergo inward and outward migrations and to accumulate around the locations of pressure maxima (Haghighipour \& Boss 2003a,b; Haghighipour 2005). 
In a gaseous disk, the turbulent eddies created by magnetorotational instability are examples of such high-pressure regions. As Johansen et al. (2006, 2007, 2008) have shown, the formation of these turbulent eddies causes small centimeter- and decimeter-sized objects to accumulate in their vicinities and increases the local density of solid material. As the accumulation of solid objects continues, their local spatial density increases until their region becomes gravitationally unstable and the accumulated bodies fragment into several 1001,000-km-sized planetesimals. This mechanism, known as streaming instability, has been presented as a scenario for planetesimal formation. [See Chiang \& Youdin (2010) for a review and Cuzzi et al. (2008) and Weidenschilling (2010) for alternative viewpoints.]

It is important to note that as shown by Shariff \& Cuzzi (2011), the local enhancement of solid to gas surface density necessary for the onset of instability is achievable only when the turbulence is extremely weak. These authors indicate that when the effect of turbulent mass diffusivity is taken into account, streaming instability becomes inefficient, and the growth rate of planetesimals reduces significantly.

Other mechanisms of the formation of planetesimals include trapping dust particles in vortices (Barge \& Sommeria 1995, Klahr \& Henning 1997, Lyra et al. 2009a), trapping particles in pressure enhanced regions created by the evaporation front of water in the protoplanetary disk (Kretke \& Lin 2007; Brauer et al. 2008a,b; Lyra et al. 2009b), turbulent concentration of solids (Chambers 2010), turbulent clustering of protoplanetary bodies (Pan et al. 2011), concentration of solid objects at the snowline (the region beyond which water is in the permanent state of ice) as a result of the sublimation of drifting ice aggregates (Aumatell \& Wurm 2011), trapping of solid objects in dead zones (Gressel et al. 2012) and at the boundary between steady super/sub-Keplerian flow created by inhomogeneous growth of magnetorotational instabilities (Kato et al. 2012), rapid coagulation of porous dust aggregates outside the snowline (Okuzumi et al. 2012), and planetesimal formation in self-gravitating disks (Gibbons et al. 2012, Shi \& Chiang 2013).

The four stages of planet formation outlined above share one interesting feature: The underlying physics of each stage is almost distinct from that of the other phases. This makes it possible to study each phase separately. Once the dust grains have grown and kilometer-sized planetesimals are formed, although the circumstellar disk still contains gas and dust, its dynamics is now mainly driven by the interaction of planetesimals with one another. These interactions are primarily gravitational, although gas drag also plays a role. At this stage, because the planetesimals are the main components populating the disk, collisions among these objects are frequent, which results in low eccentricities and low inclinations for these bodies. Because the relative velocity between two bodies is an increasing function of their orbital eccentricities, lowering the eccentricity of planetesimals due to their mutual collisions and dynamical friction, combined with their almost coplanar orbits, reduces their relative velocities. The latter facilitates the merging of these objects and enhances the rate of their accretion to larger bodies.

As a planetesimal grows, the influence zone of its gravitational field expands and as a result, it attracts more material from its surroundings. In other words, more material will be available for the planetesimal to accrete, and the rate of its growth increases. Known as runaway growth, this process results in the growth of kilometer-sized planetesimals to larger bodies in a short time (Safronov 1969; Greenberg et al. 1978; Wetherill \& Stewart 1989, 1993; Ida \& Makino 1993; 
Kokubo \& Ida 1996, 2000; Weidenschilling et al. 1997).

Runaway growth is a local process. Since the collision of two objects is more likely to result in their coalescence when their relative velocity is small, the effectiveness of this process in producing larger bodies, and the type and size of the resulting objects, varies at different distances from the central star. At large distances (e.g., > 5 AU from the Sun), where the rotational velocities are small, planetesimals approach each other with small relative velocities, and their impacts are likely to result in accretion. Also, because the temperature in the circumstellar disk is low at such distances, the bulk material of such planetesimals is primarily ice, which increases the efficiency of their sticking at the time of their collision. As a result, planetesimals at large distances grow to objects of a few Earth masses in a short time. As this process occurs while the nebular gas is still present, a growing object gradually attracts gas from its surroundings, forming a large body with a thick gaseous envelope and a mass equal to a few hundred Earth masses. At this state, a gas-giant planet is formed. This scenario, known as the core-accretion model, has been proposed as a mechanism for the formation of gas-giant planets in the Solar System (Pollack et al. 1996, Hubickyj et al. 2005, Lissauer et al. 2009, Movshovitz et al. 2010).

As the giant planets form at large orbits, the runaway accretion takes a slightly different path in the inner parts of the disk. Similar to the formation of the cores of gas-giant planets, the collisions of planetesimals at this stage may result in their growth to larger bodies. However, because the orbital motions of planetesimals are faster, they may approach each other with larger relative velocities. Also, many of these objects may lose their surface ices and other volatiles at closer distances, and as a result, when they collide with one another, the efficiency of their accretion will not be as high as for those at larger orbits. Simulations of the collision and growth of planetesimals in the inner part of the Solar System have shown that instead of forming objects as big as the cores of giant planets, accretion of these bodies results in the formation of several hundred moonto Mars-sized objects known as planetary embryos. Computational simulations (Bromley \& Kenyon 2006) and analytical analysis (Goldreich et al. 2004) have shown that when the masses of these embryos reach lunar mass, the dynamical friction of the swarm of planetesimals can no longer dampen their orbits, and their runaway growth ends. At this stage, the gravitational perturbation of the resulting planetary embryos, combined with the perturbation of giant planets, strongly affects the dynamics of smaller planetesimals and causes many of them to collide at high velocities and shatter one another, and/or their orbits become highly eccentric, and they subsequently scatter to large distances where they may leave the gravitational field of the system. This growth and clearing process continues until terrestrial planets are formed and the smaller remaining bodies (asteroids) are in stable orbits (Figure 2) (Wetherill 1990a,b, 1994, 1996; Kokubo \& Ida 1995, 1998, 2007; Chambers \& Wetherill 1998, 2001; Agnor et al. 1999; Morbidelli et al. 2000, 2012; Chambers 2001; Chambers \& Cassen 2002; Levison \& Agnor 2003; Raymond et al. 2004, 2005a,b, 2006b, 2007, 2009; Kokubo et al. 2006; OBrien et al. 2006; Hansen 2009; Schlichting et al. 2012; Torres et al. 2013; Haghighipour et al. submitted; Izidoro et al. submitted). Since the accretion and reaccretion of bodies in smaller orbits are not as efficient as in the outer regions, unlike the growth of gas-giant planets, the formation of terrestrial bodies will take several hundred million years. Figure 2 shows the time evolution of a sample simulation of terrestrial planet formation (Haghighipour et al. 
submitted; Izidoro et al. submitted). The planet formation models as explained above, although capable of explaining many features of the Solar System, face several complicated challenges. The core-accretion model, for instance, requires the nebular gas to be available for $\sim 10 \mathrm{Ma}$ while the core of Jupiter grows and accretes gas from its surroundings (Pollack et al. 1996). However, the observational estimates of the lifetimes of disks around young stars suggest a lifetime of $0.110 \mathrm{Ma}$, with $3 \mathrm{Ma}$ being the age at which half the stars show evidence of disks (Strom et al. 1993, Haisch et al. 2001, Chen \& Kamp 2004, Maercker et al. 2006). These simulations also suggest a solid core for Jupiter with a mass of $\sim 10 M_{\oplus}$. Computational modeling of the interiors of Jupiter and Saturn, however, has indicated different possible values for the cores of these objects, ranging from 0 to as large as $14 M_{\oplus}$ (Guillot 2005, Militzer et al. 2008). It is unclear what the actual masses of the cores of our gas-giant planets are, and if smaller than $10 M_{\oplus}$, how they accumulated their thick envelopes in a short time. I refer the reader to a review by Guillot (2005) for more details.

To overcome these difficulties, the core-accretion model has undergone several improvements. Hubickyj et al. (2005) and Lissauer et al. (2009) have shown that increasing the surface density of the nebula to higher than that suggested by Pollack et al. (1996) significantly reduces the time of the giant planet formation. An improved treatment of grain physics as given by Podolak (2003), Movshovitz \& Podolak (2008), and Movshovitz et al. (2010) has also indicated that the value of the grain opacity in the envelope of the growing Jupiter in the original core-accretion model (Pollack et al. 1996) is too high, and a lower value has to be adopted. This lower opacity has led to a revised version of the core-accretion model in which the time of giant planet formation is considerably smaller (Hubickyj et al. 2005, Movshovitz et al. 2010). Most recently, Bromley \& Kenyon (2011) have developed a new hybrid N-body-coagulation code that has enabled the authors to form Saturn- and Jupiter-sized planets in $\sim 1$ Ma.

An alternative model for the formation of gas-giant planets addresses this issue by proposing rapid formation of giant planets in a gravitationally unstable nebula (Boss 2000a,b, 2003; Mayer et al. 2002, 2004, 2007; Durisen et al. 2007; Boley 2009; Boley et al. 2010; Cai et al. 2010). Known as the disk-instability scenario, this model suggests that local gravitational instabilities in the solar nebula may result in the fragmentation of the disk to massive clumps that subsequently contract and form gas-giant planets in a short time. Boss's (2000a,b) and Mayer et al.'s $(2002,2003,2004)$ results show that an unstable disk can break up into giant gaseous protoplanets in as short a time as $\sim 1,000$ years. Although this mechanism presents a fast track to the formation of a gas-giant planet, it suffers from the lack of an efficient cooling process necessary to take energy away from a planet-forming clump in a sufficiently short time before it disperses.

\section{FORMATION OF SUPER-EARTHS}

The extent to which current planet formation scenarios can be used to explain the formation of super-Earths varies with the mass and orbital architecture of these objects. Since the dynamics and characteristics of planet-forming nebulae are different for stars with different spectral types, the parent stars of superEarths also play an important role. The range of masses for the currently known super-Earths, when considered within the context of giant and terrestrial planet 
formation scenarios, points to two general pathways for the formation of these objects. The low-mass super-Earths could have formed in place following a similar process as the formation of terrestrial planets in the Solar System (see, e.g., Chiang \& Laughlin 2012). The larger super-Earths, with masses close to their upper limit, may be the result of an unsuccessful and incomplete giant planet formation (see, e.g., Rogers et al. 2011). In this scenario, the super-Earths larger than terrestrial masses, combined with the fact that many of these objects are in short-period orbits, point to a formation scenario in which super-Earths are formed at large distances (where more material is available for their growth) and either migrate to their current locations as they interact with the protoplanetary disk (Kennedy \& Kenyon 2008b) or are scattered to their current orbits as a result of interactions with other cores and/or planets (Terquem \& Papaloizou 2007). In other words, the formation of these objects may have occurred while their orbital elements were evolving (Terquem \& Papaloizou 2007; Kennedy \& Kenyon 2008a,b). This mechanism naturally favors the core-accretion model of gas-giant planet formation, although attempts have also been made to explain the formation of super-Earths via the disk-instability scenario (see Section 3.3). As mentioned above, super-Earths owe their popularity to their masses and sizes, which under favorable conditions may render them habitable. While planet formation models allow for the formation of super-Earths around all types of stars (either as a failed core of a giant planet or as a slightly larger terrestrial-class object), because of the current sensitivity of detection techniques, a great deal of interest exists in super-Earths in the habitable zones of cool and low-mass stars (e.g., M dwarfs). For this reason, I devote the rest of this article to presenting a review of the models of super-Earth formation around M stars.

\subsection{Formation of Super-Earths Around Low-Mass Stars}

The discovery of planets of different sizes, from Jovian-type [e.g., GJ 876 b, c, and e (Rivera et al. 2010); HIP 57050 b (Haghighipour et al. 2010); GL 581 b (Bonfils et al. 2005); KOI-254 b (Johnson et al. 2012); Kepler-32 d (Swift et al. 2013)] to small super-Earths [e.g., GL 581 c, d, e, and g (Udry et al. 2007, Mayor et al. 2009, Vogt et al. 2010); GJ 667C c (Anglada-Escudé et al. 2011); Kepler$32 \mathrm{~b}$ and c (Swift et al. 2013)] around $\mathrm{M}$ dwarfs indicates that both giant and terrestrial planet formation can proceed efficiently around low-mass stars. This implies that the circumstellar disks around these stars can accommodate the formation of super-Earths both as a failed core of a giant planet through the gasgiant planet formation process, and also as small terrestrial-class objects through direct collisional growth of protoplanetary bodies and planetary embryos. These mechanisms have to also account for the short periods of super-Earths, whether through planet migration, planet-planet scattering, or a combination of both. I begin this section by considering the core-accretion model as the mechanism for the formation of super-Earths. As mentioned above, the discovery of superEarths can be taken as strong evidence in support of this model. However, as is explained at the end of the next section, this mechanism alone cannot explain the formation and orbital architecture of all the currently known super- Earths. Other effects such as the evolution of the central star and planet migration have to be taken into consideration as well. I discuss these effects in the next section and conclude this article by reviewing the formation of super-Earths through the disk-instability model. 


\subsection{The Core-Accretion Model}

As mentioned in Section 2, the efficiency of the core-accretion model and the rate of the growth of the cores of giant planets increase with the disk surface density. Around low-mass stars, where the surface density of the disk is smaller than around the Sun, the solid material (i.e., the planetesimals) is more spatially scattered, and as a result, the collisions among planetesimals and planetary embryos are less frequent. This smaller rate of collision prolongs the growth of planetesimals to larger sizes, and causes the time of the core growth around low-mass stars to be several times longer than the time of the formation of Jupiter around the Sun. As shown by Laughlin et al. (2004), in disks around stars with masses smaller than 0.5 solar masses $\left(M_{\odot}\right)$, the core-accretion mechanism can produce planets ranging from terrestrial-class to Neptune sizes. However, the time for the formation of these objects is much longer than the time for the formation of Jupiter in the Solar System through the core-accretion model. During this time, around M stars, for instance, the gaseous component of the circumstellar disk disperses, leaving the slowly growing core with much less gas to accrete.

The short lifetime of the gas in circumstellar disks around M stars can be attributed to two important factors:

- the high internal radiation of young M stars (at this stage, these stars are almost as bright as Sun-like stars), and

- external perturbations from other close-by stars.

The latter is primarily due to the fact that most stars are formed in clusters (Lada \& Lada 2003), and as such, their circumstellar disks are strongly affected by the gravitational perturbations and the radiations of other stars (Adams et al. 2004). For M stars, this causes the circumstellar disk to receive a high amount of radiation from both the central star and external sources. This high amount of radiation combined with the low masses of $\mathrm{M}$ stars, which points to their small gravitational fields, increases the effectiveness of the photoevaporation of the gaseous component of the circumstellar disk by up to two orders of magnitude. As a result, the majority of the gas leaves the disk at the early stages of giant planet formation, leaving a still-forming core with not much gas to accrete.

\subsubsection{EFFECT OF STELLAR EVOLUTION}

Although the growth of giant planets cores through collision and accretion of planetesimals is similar in disks around solar-type and low-mass stars, the fact that around smaller stars this process takes longer introduces a fundamental difference in the formation of giant planets in these two environments. As opposed to young Sun-like stars whose luminosities stay almost constant during the formation of giant and terrestrial planets (e.g., 10-100 Ma), the luminosity of a premain sequence, low-mass star (e.g., $0.5 M_{\odot}$ ) fades by a factor of 10 to 100 during this process (Hayashi 1981). This causes the internal temperature of the circumstellar disk to decrease, which subsequently causes the disks snowline to move toward the central star and to close distances. The forward migration of the snowline results in an increase in the population of icy materials (kilometer-sized and larger planetesimals) in the outer regions of the disk, which in turn increases the efficiency of the collisional growth of these objects to protoplanetary bodies (as mentioned in Section 2, sticking is more efficient among icy bodies). As shown by Kennedy et al. (2006), around a $0.25-M_{\odot}$ star, the moving snowline 
causes rapid formation of planetary embryos within a few million years (also see Kennedy et al. 2007). Subsequent collisions and interactions among these objects result in the formation of super-Earths in approximately 50-500 Ma.

\subsubsection{EFFECT OF PLANET MigRATION}

As mentioned above, one of the major developments in the field of planetary dynamics that was a direct consequence of the detection of extrasolar planets is the concept of planet migration. Although previously post-formation migration had been proposed as a mechanism to explain the orbital architecture of small bodies in the Solar System (e.g., moons of giant planets and Kuiper belt objects), the migration of planets during their formation had not been incorporated into the models of planetary formation. In other words, the planet formation scenarios mentioned above were developed assuming that planets form in place. The discovery of extrasolar planets, almost from the beginning, challenged this assumption. The detection of the first hot Jupiter in a 4-day orbit around the star 51 Pegasi (Mayor \& Queloz 1995) revealed that planet migration is an inseparable part of the evolution of a planetary system and prompted astronomers to revisit this concept and to incorporate it into their models of planet formation. Today, planet migration is well developed and widely accepted as part of a comprehensive planet formation scenario.

Planetary and satellite migration has long been recognized as a major contributor to the formation and orbital architecture of planets, their moons, and other minor bodies in the Solar System. As shown by Greenberg et al. (1972) and Greenberg (1973), mean-motion resonances (i.e., commensurable orbital periods6) among the natural satellites of giant planets (e.g., Titan and Hyperion, satellites of Saturn) may have been the result of the radial migration of these objects due to their tidal interactions with their parent planets (Goldreich 1965). The dynamical architecture of Galilean satellites, with their three-body, Laplace resonance, has also been attributed to the migration of these objects. It is accepted that these satellites migrated inward during their formation as a result of interacting with the circumplanetary disk of satellitesimals around Jupiter (Canup \& Ward 2002), and subsequently by tidal forces after their formation (Peale \& Lee 2002). The lack of irregular satellites between Callisto, the outermost Galilean satellite, and Themisto, the innermost irregular satellite of Jupiter, also can be explained by a dynamical clearing process that occurred during the formation and migration of Galilean satellites (Haghighipour \& Jewitt 2008). Among the planets of our Solar System, the post-formation, planetesimal-driven migration of giant planets has been proposed as a mechanism to explain the current state of the asteroid belt (Tsiganis et al. 2005; Minton \& Malhotra 2009, 2011; see also Gomes 1997), late heavy bombardment (Gomes et al. 2005), the origin of Jupiter Trojan asteroids (Morbidelli et al. 2005), the effects of secular resonances on terrestrial planet formation (Agnor \& Lin 2012), and the small mass and size of Mars (Walsh et al. 2011). I refer the reader to Morbidelli et al. (2012) for a review on these topics.

The idea of the migration of planetary bodies was first proposed by Fernandez

\footnotetext{
${ }^{6}$ Orbital commensurability is necessary for two planets to be in a mean-motion resonance; however, it is not sufficient. Other constraints have to exist between the angular elements of their orbits as well. For more details, the reader is referred to Roy (1982), Danby (1992), and Murray \& Dermott (1999).
} 
\& Ip (1984). These authors suggested that after the dispersal of the nebular gas, fully formed giant planets may drift from their original orbits due to the exchange of angular momentum with the disk of planetesimals. As a result of this post-formation migration, small bodies either are scattered out of the Solar System or may reach other regions where they may reside in long-term stable orbits. As shown by Malhotra (1993, 1995), this mechanism can explain the peculiar orbit of Pluto (highly eccentric, inclined, and long-term chaotic), and as shown by Malhotra (1996) and Hahn \& Malhotra (2005), it can also explain the dynamical structure of Kuiper belt objects.

The past two decades have witnessed major developments in the theories of planet migration. Simulations of the formation of planetary bodies and their interactions with circumstellar disks have shown that planet migration does not have to occur necessarily after the planets are fully formed. In fact, planets can migrate while they are forming as a result of exchanging angular momentum with their surrounding environment. This naturally suggests that the physical and dynamical characteristics of a planet and its circumstellar disk will play an important role in this process. For instance, the planet may undergo type I migration, in which case it does not accrete nebular material as it migrates (Figure 3a). Conversely, the planet may be large and accrete nebular material, in which case it may create a gap in the disk as it migrates (Figure 3b). This type of migration is known as type II migration. Planet migration may occur in other forms as well. 7

The contribution of planet migration to the formation of close-in super-Earths may appear in different forms. The most common scenario involves the inward migration of a fully formed giant planet in a disk of planetesimals and planetary embryos. The giant planet in this scenario affects the dynamics of protoplanetary bodies interior to its orbit by either increasing their orbital eccentricities and scattering them to larger distances or causing them to migrate to closer orbits. The migrating protoplanets may be shepherded by the giant planet into small close-in regions, where they are captured in mean-motion resonances. As Zhou et al. (2005), Fogg \& Nelson (2005, 2006, 2007a,b, 2009), and Raymond et al. (2008) have shown, around Sun-like stars, the shepherded protoplanets may also collide and grow to terrestrial-class and super-Earth objects (see, e.g., Figure 6b). Studies of the back-scattered objects in the simulations of disks around massive stars have shown that these bodies may also collide and grow to planetary sizes (Mandell \& Sigurdsson 2003, Raymond et al. 2006a, Mandell et al. 2007).

While around Sun-like stars, despite the out-scattering of protoplanetary bodies during the migration of a giant planet, the formation of super-Earths through the collision and growth of planetesimals and planetary embryos proceeds efficiently, around low-mass stars this scenario is not always the case. Simulations of the dynamics of protoplanetary bodies at distances smaller than $0.2 \mathrm{AU}$ around a $0.3 M_{\odot}$ star have shown that during the inward migration of one or several giant planets (the latter involves migrating planets in mean-motion resonances), the majority of the protoplanets leave the system and do not contribute to the forma-

\footnotetext{
${ }^{7}$ I do not discuss these mechanisms here, as they may not be entirely relevant to the formation and dynamical evolution of super-Earths. Instead, I refer the reader to numerous articles that have been published on these subjects. Unfortunately, the richness of the literature does not allow me to cite all these articles here, but among them, one can refer to Nelson et al. (2001), Massét \& Snellgrove (2001), Papaloizou \& Terquem (2006), Chambers (2009), Armitage (2010), and a recent review by Baruteau \& Massét (2013).
} 
tion of close-in Earth-sized bodies and/or super-Earths (Figure 4) (Haghighipour \& Rastegar 2011). These results suggest that the currently known small planets around M stars might have formed at larger distances and were either scattered to their current close-in orbits (e.g., GJ 876 d; see Figure 5) or migrated into their orbits while captured in a mean-motion resonance with a migrating planet.

The above-mentioned scenario for the formation of close-in super-Earths is based on the fact that giant planets are formed long before the protoplanetary bodies grow to larger sizes. The underlying assumption in this scenario is that the giant planet does not migrate during its formation, and the migration of the planetary embryos (the moon- to Mars-sized objects) is also ignored. However, not only do the cores of still-forming giant planets migrate (Alibert et al. 2004), so too do the planetary embryos. While migrating, the embryos may undergo orbital crossing and collisional merging, which may result in their growth to a few super-Earths, especially in mean motion resonances. Simulating the interactions of 25 protoplanetary objects with masses ranging from 0.1 to $1 M_{\oplus}$, Terquem \& Papaloizou (2007) have shown that a few close-in super-Earths may form in this way with masses up to $12 M_{\oplus}$. The results of these simulations suggest that in systems in which merging of migrating cores results in the formation of superEarths and Neptune-like planets, such planets will always be accompanied by giant bodies and most likely will be in mean-motion resonances. Similar results have also been reported by Haghighipour \& Rastegar (2011).

Interestingly, several planetary systems have been discovered in which central stars host only small Neptune-sized objects and super-Earths (e.g., HD 69830, GL 581). The planets in these systems do not have a Jupiter-like companion that could have migrated to facilitate their formation. Such systems seem to imply that a different mechanism may be responsible for the formation of their super-Earth bodies. Kennedy \& Kenyon (2008a) and Kenyon \& Bromley (2009) have suggested that the migration of protoplanetary embryos may be the key in facilitating the close-in accretion of these objects. These authors considered a circumstellar disk with a density enhancement at the region of its snowline and simulated the dynamics and growth of its planetary embryos. They showed that while interacting with one another (colliding and accreting), many of these objects may migrate toward the central star. Around a solar-type star, the time of such migrations for an Earth-sized planet at $1 \mathrm{AU}$ is $\sim 10^{5}-10^{6}$ years - much smaller than the time for the chaotic growth of a typical moon- or Mars-sized embryo $\left(10^{8}\right.$ years) (Goldreich et al. 2004). This implies that most of the migration occurs prior to the onset of the final growth. Depending on their relative velocities, the interactions among the migrating embryos may result in their growth, scattering, and/or shepherding, as in the case of a migrating giant planet. Simulations by Kennedy \& Kenyon (2008b) and Kenyon \& Bromley (2009) have shown that super-Earth objects with masses up to $8 M_{\oplus}$ may form in this way around stars ranging from 0.25 to $2 M_{\odot}$ (Figure 6$)$.

\subsection{The Disk-Instability Model}

The formation of super-Earths through the mechanisms explained above, particularly when those mechanisms are used to explain the formation of these objects at the higher end of their mass range, naturally favors the core-accretion model of giant planet formation. However, the fact that Jovian-type planets have been discovered around low-mass stars (e.g., GJ 876, with three planets ranging from 
1 Uranus mass to 2.2 Jupiter masses in $~ 120-, 60$-, and 30-day orbits; HIP57050, with a Saturn-mass planet in a $\sim 40$-day orbit) suggests that the disk-instability model may also be able to form close-in super-Earths, especially those that are considered as failed cores of giant planets. As explained above, given the low masses of the circumstellar disks around $M$ stars, the existence of giant planets around these stars suggests that they might have formed at large distances and migrated to their current orbits. This is because in a planet-forming nebula, more nebular material is available at outer regions that can then facilitate the formation of a giant planet through the core-accretion model. The availability of more mass at outer distances in a disk may also trigger the formation of giant planets around $\mathrm{M}$ stars through the disk-instability scenario. Recall that in this scenario, clumps, formed in an unstable gaseous disk, collapse and form gas-giant planets (e.g., Boss 2000b, Mayer et al. 2002). After the giant planets are formed, a secondary process is needed to remove their gaseous envelopes. As Boss (2006) has shown, such collapsing clumps can form around a $0.5 M_{\odot}$ star at a distance of $\sim 8 \mathrm{AU}$ (Figure 7). This author suggests that, as most stars are formed in clusters and in high-mass, star-forming regions, intense far/extreme UV radiations from nearby $\mathrm{O}$ stars may rapidly (within $1 \mathrm{Ma}$ ) photoevaporate the gaseous envelopes around giant planets, leaving them with large super-Earth cores. Similar mechanisms have been suggested for the formation of Uranus and Neptune in the Solar System (Boss et al. 2002). A subsequent migration, similar to that suggested by Michael et al. (2011), may then move these cores to close-in orbits.

\section{CONCLUDING REMARKS}

As evident from this review, it is generally accepted that super-Earths are formed through a combination of a core accumulation process and planetary migration. Modeling the formation of these objects requires the simulation of the collisional growth of planetary embryos and their subsequent interactions with the protoplanetary disk. A realistic model requires global treatment of the disk and inclusion of large numbers of planetesimals and planetary embryos. In practice, such simulations are computationally expensive. To avoid such complications, most of the current models of super-Earth formation include only small numbers of objects (e.g., cores, progenitors, protoplanets, planetesimals). As shown by McNeil \& Nelson (2010), in systems with large numbers of bodies (e.g., several thousand planetesimals and larger objects), the combination of traditional core accretion and type I planet migration may not produce objects larger than 3-4 $M_{\oplus}$ in close-in (e.g., $\leq 0.5 \mathrm{AU}$ ) orbits. Although the systems studied carry some simplifying assumption, McNeil \& Nelsons results point to an interesting conclusion: While the combination of core accretion and planet migration seems to be a viable mechanism for the formation of close-in super-Earths, the formation of these objects is still an open question, and a comprehensive theory for their formation requires more sophisticated computational modeling, with possibly entirely new physics, as yet to be discovered.

\section{ACKNOWLEDGMENT}

I am grateful to Jörgen Blum, Alan Boss, Andre Izidoro, Scott Kenyon, Frédéric Massét, and Ji-Lin Zhou for kindly providing figures. 


\section{REFERENCES}

Adams FC, Hollenbach D, Laughlin G, GortiU. 2004. Photoevaporation of circumstellar disks due to external far-ultraviolet radiation in stellar aggregates. Astrophys. J. 611:360-79

Agnor CB, Canup RM, Levison HF. 1999. On the character and consequences of large impacts in the late stage of terrestrial planet formation. Icarus 142:219-37

Agnor CB, Lin DNC. 2012. On the migration of Jupiter and Saturn: constraints from linear models of secular resonant coupling with the terrestrial planets. Astrophys. J. 745:143

Alibert Y, Mordasini C, Benz W. 2004. Migration and giant planet formation. Astron. Astrophys. 417:L25-28

Anglada-Escudé G, Arriagad P, Vogt SS, Rivera E, Butler RP. 2011. A planetary system around the nearby M dwarf GJ $667 \mathrm{C}$ with at least one super-Earth in its habitable zone. Astrophys. J. 751:L16

Armitage PJ. 2010. The early evolution of planetary systems. In Astrophysics of Planet Formation, pp. 218-62. Cambridge, UK: Cambridge Univ. Press

Aumatell G, Wurm G. 2011. Breaking the ice: planetesimal formation at the snowline. MNRAS 418:L1-5

Barge P, Sommeria J. 1995. Did planet formation begin inside persistent gaseous vortices? Astron. Astrophys. 295:L1-4

Baruteau C, Masset F. 2013. Recent developments in planet migration theory. In Tides in Astronomy and Astrophysics (Lecture Notes in Physics), ed. J Souchay, S Mathis, T Tokieda, p. 201. Berlin: Springer-Verlag

Bean JL, Désert J-M, Kabath P, Stalder B, Seager S, et al. 2011. The optical and near-infrared transmission spectrum of the super-Earth GJ 1214b: further evidence for a metal-rich atmosphere. Astrophys. J. 743:92

Bean JL, Miller-Ricci Kempton E, Homeier D. 2010. A ground-based transmission spectrum of the super-Earth exoplanet GJ 1214b. Nature 468:669-72

Beitz E, Güttler C, Blum J, Meisner T, Teiser J,Wurm G. 2011. Low-velocity collisions of centimeter-sized dust aggregates. Astrophys. J. 736:34

Berta ZK, Charbonneau D, Désert J-M, Miller-Ricci Kempton E, McCullough PR, et al. 2012. The flat transmission spectrum of the super-Earth GJ1214b from Wide Field Camera 3 on the Hubble Space Telescope. Astrophys. J. 747:35

Blum J. 2006. Dust agglomeration. Adv. Phys. 55:881-947 
Blum J. 2010. Dust growth in protoplanetary disksa comprehensive experimental/theoretical approach. Res. Astron. Astrophys. 10:1199-214

Blum J, Münch M. 1993. Experimental investigations on aggregate-aggregate collisions in the early solar nebula. Icarus 106:151-67

Blum J, Wurm G. 2000. Experiments on sticking, restructuring, and fragmentation of preplanetary dust aggregates. Icarus 143:138-46

Blum J, Wurm G. 2008. The growth mechanisms of macroscopic bodies in protoplanetary disks. Annu. Rev. Astron. Astrophys. 46:21-56

Blum J, Wurm G, Poppe T, Heim L-O. 1998. Aspects of laboratory dust aggregation with relevance to the formation of planetesimals. Earth Moon Planets $80: 285-309$

Boley AC. 2009. The two modes of gas-giant planet formation. Astrophys. J. 695:L53-57

Boley AC, Hayfield T,Mayer L, Durisen RH. 2010. Clumps in the outer disk by disk instability: why they are initially gas giants and the legacy of disruption. Icarus 207:509-16

Bonfils X, Forveille T, Delfosse X, Udry S, Mayor M. 2005. The HARPS search for southern extra-solar planets. VI. A Neptune-mass planet around the nearby M dwarf Gl 581. Astron. Astrophys. 443:L15-18

Boss AP. 2000a. Formation of extrasolar giant planets: core accretion or disk instability? Earth Moon Planets 81:19-26

Boss AP. 2000b. Possible rapid gas-giant planet formation in the solar nebula and other protoplanetary disks. Astrophys. J. 536:L101-4

Boss AP. 2003. Rapid formation of outer giant planets by disk instability. Astrophys. J. 599:577-81

Boss AP. 2006. Rapid formation of super-Earths around M dwarf stars. Astrophys. J. 644:L79-82

Boss AP,Wetherill GW, Haghighipour N. 2002. Rapid formation of ice giant planets. Icarus 156:291-95

Brauer F, Dullemond CP, Henning T. 2008a. Coagulation, fragmentation and radial motion of solid particles in protoplanetary disks. Astron. Astrophys. 480:859-77

Brauer F, Henning T, Dullemond CP. 2008b. Planetesimal formation near the snow line in MRI-driven turbulent protoplanetary disks. Astron. Astrophys. 487:L1-4 
Bromley BC, Kenyon SJ. 2006. Terrestrial planet formation. I. The transition from oligarchic growth to chaotic growth. Astron. J. 131:1837-50

Bromley BC, Kenyon SJ. 2011. A new hybrid N-body-coagulation code for the formation of gas giant planets. Astrophys. J. 731:101

Cai K, Pickett MK, Durisen RH, Milne AM. 2010. Giant planet formation by disk instability: a comparison simulation with an improved radiative scheme. Astrophys. J. 716:L176-80

Canup RM, Ward WR. 2002. Formation of the Galilean satellites: conditions of accretion. Astron. J. 124:3404-23

Chambers JE. 2001. Making more terrestrial planets. Icarus 152:205-24

Chambers JE. 2009. Planetary migration: What does it mean for planet formation? Annu. Rev. Earth Planet. Sci. 37:321-44

Chambers JE. 2010. Planetesimal formation by turbulent concentration. Icarus 208:505-17

Chambers JE, Cassen P. 2002. The effect of surface density profile and giant planet eccentricities on planetary accretion in the inner solar system. Meteorit. Planet. Sci. 37:1523-40

Chambers JE, Wetherill GW. 1998. Making the terrestrial planets: N-body integrations of planetary embryos in three dimensions. Icarus 136:304-27

Chambers JE, Wetherill GW. 2001. Planets in the asteroid belt. Meteorit. Planet. Sci. 36:38199 Charbonneau D, Berta ZK, Irwin J, Burke CJ, Nutzman P, et al. 2009. A super-Earth transiting a nearby low-mass star. Nature 462:891-94

Chen CH, Kamp I. 2004. Are giant planets forming around HR 4796A? Astrophys. J. 602:985-92

Chiang E, Laughlin G. 2013. The minimum-mass extrasolar nebula: in-situ formation of close-in super-Earths. MNRAS 413:3444-55

Chiang E, Youdin AN. 2010. Forming planetesimals in solar and extrasolar nebulae. Annu. Rev. Earth Planet. Sci. 38:493-522

Clubb K, Fischer D, Howard A, Marcy G, Henry G. 2009. M2K: a search for planets orbiting early M and late K dwarf stars. Bull. Am. Astron. Soc. 41:192

Cuzzi JN, Hogan RC, Shariff K. 2008. Towards planetesimals: dense chondrule clumps in the protoplanetary nebula. Astrophys. J. 687:1432-47 
Danby JMA. 1992. Fundamentals of Celestial Mechanics. Richmond, VA: WillmannBell. 2nd ed.

Désert J-M, Bean J, Miller-Ricci Kempton E, Berta ZK, Charbonneau D, et al. 2011. Observational evidence for a metal-rich atmosphere on the super-Earth GJ1214b. Astrophys. J. 731:L40

Dominik C, Tielens AGGM. 1997. The physics of dust coagulation and the structure of dust aggregates in space. Astrophys. J. 480:647-73

Dressing CD, Charbonneau D. 2013. The occurrence rate of small planets around small stars. Astrophys. J. 767:95

Dullemond CP, Dominik C. 2005. Dust coagulation in protoplanetary disks: a rapid depletion of small grains. Astron. Astrophys. 434:971-86

Durisen RH, Boss AP, Mayer L, Nelson AF, Quinn T, Rice WKM. 2007. Gravitational instabilities in gaseous protoplanetary disks and implications for giant planet formation. In Protostars and PlanetsV, ed. B Reipurth, D Jewitt, K Keil, pp. 607-22. Tucson: Univ. Ariz. Press

Fernandez JA, Ip W-H. 1984. Some dynamical aspects of the accretion of Uranus and Neptune-the exchange of orbital angular momentum with planetesimals. Icarus 58:109-20

Fogg MJ, Nelson RP. 2005. Oligarchic and giant impact growth of terrestrial planets in the presence of gas-giant planet migration. Astron. Astrophys. 441:791-806

Fogg MJ, Nelson RP. 2006. On the possibility of terrestrial planet formation in hot-Jupiter systems. Int. J. Astrobiol. 5:199-209

Fogg MJ, Nelson RP. 2007a. On the formation of terrestrial planets in hot-Jupiter systems. Astron. Astrophys. 461:1195-208

Fogg MJ, NelsonRP. 2007b. The effect of type I migration on the formation of terrestrial planets in hot-Jupiter systems. Astron. Astrophys. 472:1003-15

Fogg MJ, Nelson RP. 2009. Terrestrial planet formation in low-eccentricity warmJupiter systems. Astron. Astrophys. 498:575-89

Forveille T, Bonfils X, Delfosse X, Alonso R, Udry S, et al. 2011. The HARPS search for southern extrasolar planets. XXXII. Only 4 planets in GL 581 system. arXiv:1109.2505, http://arxiv.org/abs/1109.2505

Fraine JD, Deming D, Gillon M, Jehin E, Demory B-O, et al. 2013. Spitzer transits of the super-Earth GJ $1214 \mathrm{~b}$ and implications for its atmosphere. Astrophys. J. $765: 127$

Gibbons PG, Rice WKM, Mamatsashvili GR. 2012. Planetesimal formation in 
self-gravitating discs. MNRAS 426:1444-54

Goldreich P. 1965. An explanation of the frequent occurrence of commensurable mean motions in the Solar System. MNRAS 130:159-81

Goldreich P, Lithwick Y, Sari R. 2004. Final stages of planet formation. Astrophys. J. 614:497-507

Gomes R, Levison HF, Tsiganis K, Morbidelli A. 2005. Origin of the cataclysmic Late Heavy Bombardment period of the terrestrial planets. Nature 435:466-69

Gomes RS. 1997. Dynamical effects of planetary migration on the primordial asteroid belt. Astron. J. 114:396-401

Greenberg RJ. 1973. Evolution of satellite resonances by tidal dissipation. Astron. J. 78:338-46

Greenberg RJ, Counselman CC, Shapiro II. 1972. Orbit-orbit resonance capture in the Solar System. Science 178:747-49

Greenberg R, Hartmann WK, Chapman CR, Wacker JF. 1978. Planetesimals to planets-numerical simulations of collisional evolution. Icarus 35:1-26

Gressel O, Nelson RP, Turner NJ. 2012. Dead zones as safe havens for planetesimals: influence of disc mass and external magnetic field. MNRAS 422:1140-59

Guillot T. 2005. The interiors of giant planets: models and outstanding questions. Annu. Rev. Earth Planet. Sci. 33:493-530

Güttler C, Blum J, Zsom A, Ormel CW, Dullemond CP. 2009. The first phase of protoplanetary dust growth: the bouncing barrier. Geochim. Cosmochim. Acta 73:A482

Haghighipour N. 2005. Growth and sedimentation of dust particles in the vicinity of local pressure enhancements in a solar nebula. MNRAS 362:1015-24

Haghighipour N. 2011. Super-Earths: a new class of planetary bodies. Contemp. Phys. 52:403-38

Haghighipour N, Boss AP. 2003a. On pressure gradients and rapid migration of solids in a nonuniform solar nebula. Astrophys. J. 583:996-1003

Haghighipour N, Boss AP. 2003b. On gas draginduced rapid migration of solids in a nonuniform solar nebula. Astrophys. J. 598:1301-11

Haghighipour N, Jewitt D. 2008. A region void of irregular satellites around Jupiter. Astron. J. 136:909-18

Haghighipour N, Rastegar S. 2011. Implications of the TTV-detection of close- 
in terrestrial planets around $\mathrm{M}$ stars for their origin and dynamical evolution. Proc. Haute-Provence Obs. Colloq., St. Michel l'Observatoire, France, Aug. 2327, 2010, ed. F Bouchy, RF Diaz, C Moutou, 11:04004. Les Ulis, France: EPJ Web of Conferences. http://dx.doi.org/10.1051/epjconf/20101104004

Haghighipour N, Vogt SS, Butler RP, Rivera EJ, Laughlin G, et al. 2010. The Lick-Carnegie Exoplanet Survey: a Saturn-mass planet in the habitable zone of the nearby M4V star HIP 57050. Astrophys. J. 715:271-76

Hahn JM, Malhotra R. 2005. Neptunes migration into a stirred-up Kuiper belt: a detailed comparison of simulations to observations. Astron. J. 130:2392-414

Haisch KE Jr, Lada EA, Lada CJ. 2001. Disk frequencies and lifetimes in young clusters. Astrophys. J. Lett. 553:L153-56

Hansen BMS. 2009. Formation of the terrestrial planets from a narrow annulus. Astrophys. J. 703:1131-40

Hatzes AP, Dvorak R, Wuchterl G, Guterman P, Hartmann M, et al. 2010. An investigation into the radial velocity variations of CoRoT-7. Astron. Astrophys. 520:93-108

Hatzes AP, Fridlund M, Nachmani G, Mazeh T, Valencia D, et al. 2011. The mass of CoRoT-7 b. Astrophys. J. 743:75

Hayashi C. 1981. Structure of the solar nebula, growth and decay of magnetic fields and effects of magnetic and turbulent viscosities on the nebula. Prog. Theor. Phys. 70:S35-53

Heng K, Vogt SS. 2011. Gliese 581g as a scaled-up version of Earth: atmospheric circulation simulations. MNRAS 415:2145-57

Hubickyj O, Bodenheimer P, Lissauer JJ. 2005. Accretion of the gaseous envelope of Jupiter around a 5-10 Earth-mass core. Icarus 179:415-31

Ida S, Makino J. 1993. Scattering of planetesimals by a protoplanet: slowing down of runaway growth. Icarus 106:210-27

Irwin J, Charbonneau D, Nutzman P, Falco E. 2009a. The MEarth Project: searching for transiting habitable super-Earth planets around nearby M-dwarfs. Proc. 15th Cambridge Workshop Cool Stars Stellar Syst. Sun, St. Andrews, Scotl., July 21-25, 2008. AIP Conf. Proc. 1094:445-48. Melville, NY: AIP

Irwin J, Charbonneau D, Nutzman P, Falco E. 2009b. The MEarth Project: searching for transiting habitable super-Earths around nearby M-dwarfs. Proc. Int. Astron. Union Symp., Boston, May 19-23, 2008. 253:37-43. Cambridge, UK: Cambridge Univ. Press

Johansen A, Brauer F, Dullemond C, Klahr H, Henning T. 2008. A coagulation- 
fragmentation model for the turbulent growth and destruction of preplanetesimals. Astron. Astrophys. 486:597-611

Johansen A, Klahr H,Henning T. 2006. Gravoturbulent formation of planetesimals. Astrophys. J. 636:1121-34

Johansen A, Oishi JS, Mac Low M-M, Klahr H, Henning T, Youdin A. 2007. Rapid planetesimal formation in turbulent circumstellar disks. Nature 448:102225

Johnson JA, Gazak JZ, Apps K, Muirhead PS, Crepp JR, et al. 2012. Characterizing the cool KOIs. II. The M dwarf KOI-254 and its hot Jupiter. Astron. J. $143: 111$

Kato MT, Fujimoto M, Ida S. 2012. Planetesimal formation at the boundary between steady super/sub-Keplerian flow created by inhomogeneous growth of magnetorotational instability. Astrophys. J. 747:11-20

Kennedy GM, Kenyon SJ. 2008a. Planet formation around stars of various masses: the snow line and the frequency of giant planets. Astrophys. J. 673:50212

Kennedy GM, Kenyon SJ. 2008b. Planet formation around stars of various masses: hot super-Earths. Astrophys. J. 682:1264-76

Kennedy GM, Kenyon SJ, Bromley BC. 2006. Planet formation around low-mass stars: the moving snow line and super-Earths. Astrophys. J. 650:L139-42

Kennedy GM, Kenyon SJ, Bromley BC. 2007. Planet formation around Mdwarfs: the moving snow line and super-Earths. Astrophys. Space Sci. 311:9-13

Kenyon SJ, Bromley BC. 2009. Rapid formation of icy super-Earths and the cores of gas-giant planets. Astrophys. J. 690:L140-43

Klahr HH, Henning T. 1997. Particle-trapping eddies in protoplanetary accretion disks. Icarus 128:213-29

Kokubo E, Ida S. 1995. Orbital evolution of protoplanets embedded in a swarm of planetesimals. Icarus 114:247-57

Kokubo E, Ida S. 1996. On runaway growth of planetesimals. Icarus 123:180-91

Kokubo E, Ida S. 1998. Orbital oligarchic growth of protoplanets. Icarus 131:17178

Kokubo E, Ida S. 2000. Formation of protoplanets from planetesimals in the solar nebula. Icarus 143:15-27

Kokubo E, Ida S. 2007. Formation of terrestrial planets from protoplanets. II. Statistics of planetary spin. Astrophys. J. 671:2082-90 
Kokubo E, Kominami J, Ida S. 2006. Formation of terrestrial planets from protoplanets. I. Statistics of basic dynamical properties. Astrophys. J. 642:1131-39

Krause M, Blum J. 2004. Growth and form of planetary seedlings: results from a sounding rocket microgravity aggregation experiment. Phys. Rev. Lett. 93:021103

Kretke KA, Lin DNC. 2007. Grain retention and formation of planetesimals near the snow line inMRI-driven turbulent protoplanetary disks. Astrophys. J. 664:L55-58

Lada CJ, Lada EA. 2003. Embedded clusters in molecular clouds. Annu. Rev. Astron. Astrophys. 41:57-115

Laughlin G, Bodenheimer P, Adams FC. 2004. The core-accretion model predicts few Jovian-mass planets orbiting red dwarfs. Astrophys. J. 612:L73-76

Léger A, Rouan D, Schneider J, Barge P, Fridlund M, et al. 2009. Transiting exoplanets from the CoRoT space mission. VIII. CoRoT-7b: the first super-Earth with measured radius. Astron. Astrophys. 506:287-302

Levison HF, Agnor C. 2003. The role of giant planets in terrestrial planet formation. Astron. J. 125:2692-713

Lissauer JJ, Hubickyj O, D'Angelo G, Bodenheimer P. 2009. Models of Jupiters growth incorporating thermal and hydrodynamic constraints. Icarus 199:338-50

Lyra W, Johansen A, Klahr H, Piskunov N. 2009a. Standing on the shoulders of giants. Trojan earths and vortex trapping in low mass self-gravitating protoplanetary disks of gas and solids. Astron. Astrophys. 493:1125-39

Lyra W, Johansen A, Zsom A, Klahr H, Piskunov N. 2009b. Planet formation bursts at the borders of the dead zone in 2D numerical simulations of circumstellar disks. Astron. Astrophys. 497:869-88

Maercker M, Burton MG, Right WCM. 2006. L-band (3.5 m) IR-excess in massive star formation. II. RCW 57/NGC 3576. Astron. Astrophys. 450:253-63

Malhotra R. 1993. The origin of Plutos peculiar orbit. Nature 365:819-21

Malhotra R. 1995. The origin of Plutos orbit: implications for the Solar System beyond Neptune. Astron. J. 110:420-29

Malhotra R. 1996. The phase space structure near Neptune resonances in the Kuiper belt. Astron. J. 111:504-16

Mandell AM, Raymond SN, Sigurdsson S. 2007. Formation of Earth-like planets during and after giant planet migration. Astrophys. J. 660:823-44 
Mandell AM, Sigurdsson S. 2003. Survival of terrestrial planets in the presence of giant planet migration. Astrophys. J. 599:L111-14

Massét F, Snellgrove M. 2001. Reversing type II migration: resonance trapping of a lighter giant protoplanet. MNRAS 320:L55-59

Mayer L, Lufkin G, Quinn T, Wadsley J. 2007. Fragmentation of gravitationally unstable gaseous protoplanetary disks with radiative transfer. Astrophys. J. 661:L77-80

Mayer L, Quinn T, Wadsley J, Stadel J. 2002. Formation of giant planets by fragmentation of protoplanetary disks. Science 298:1756-59

Mayer L, Quinn T, Wadsley J, Stadel J. 2003. Simulations of unstable gaseous disks and the origin of giant planets. In ASP Conference Series. Vol. 294: Scientific Frontiers in Research on Extrasolar Planets, ed. D Deming, S Seager, pp. 28186. San Francisco: Astron. Soc. Pac.

Mayer L, Quinn T, Wadsley J, Stadel J. 2004. The evolution of gravitationally unstable protoplanetary disks: fragmentation and possible giant planet formation. Astrophys. J. 609:1045-64

MayorM, Bonfils X, Forveille T, Delfosse X, Udry S, et al. 2009. The HARPS search for southern extrasolar planets. XVIII. An Earth-mass planet in the GJ 581 planetary system. Astron. Astrophys. 507:487-94

Mayor M, Queloz D. 1995. A Jupiter-mass companion to a solar-type star. Nature $378: 355-59$

McNeil DS, Nelson RP. 2010. On the formation of hot Neptunes and superEarths. MNRAS 401:1691-708

Menou K. 2012. Atmospheric circulation and composition of GJ 1214 b. Astrophys. J. 744:L16

Michael S, Durisen RH, Boley AC. 2011. Migration of gas giant planets in gravitationally unstable disks. Astrophys. J. 737:L42

Militzer B, Hubbard WB, Vorberger J, Tamblyn I, Bonev SA. 2008. A massive core in Jupiter predicted from first-principles simulations. Astrophys. J. 688:L4548

Miller-Ricci E, Fortney JJ. 2010. The nature of the atmosphere of the transiting super-Earth GJ 1214 b. Astrophys. J. Lett. 716:L74-79

Miller-Ricci E, Seager S, Sasselov D. 2009. The atmospheric signatures of superEarths: how to distinguish between hydrogen-rich and hydrogen-poor atmospheres. Astrophys. J. 690:1056-67 
Minton DA, Malhotra R. 2009. A record of planet migration in the main asteroid belt. Nature 457:1109-11

Minton DA, Malhotra R. 2011. Secular resonance sweeping of the main asteroid belt during planet migration. Astrophys. J. 732:53

Mizuno H, Markiewicz WJ, Völk HJ. 1988. Grain growth in turbulent protoplanetary accretion disks. Astron. Astrophys. 195:183-92

Morbidelli A, Chambers J, Lunine JL, Petit JM, Robert F, et al. 2000. Source regions and timescales for the delivery of water to Earth. Meteorit. Planet. Sci. $35: 1309-20$

Morbidelli A, Levison HF, Tsiganis K, Gomes R. 2005. Chaotic capture of Jupiters Trojan asteroids in the early Solar System. Nature 435:462-65

Morbidelli A, Lunine JI, O'Brien DP, Raymond SN, Walsh KJ. 2012. Building terrestrial planets. Annu. Rev. Earth Planet. Sci. 40:251-75

Movshovitz N, Bodenheimer P, Podolak M, Lissauer JJ. 2010. Formation of Jupiter using opacities based on detailed grain physics. Icarus 209:616-24

Movshovitz N, Podolak M. 2008. The opacity of grains in protoplanetary atmospheres. Icarus 194:368-78

Murray CD, Dermott SF. 1999. Solar System Dynamics. Cambridge, UK: Cambridge Univ. Press

Nakagawa Y, Nakazawa K, Hayashi C. 1981. Growth and sedimentation of dust grains in the primordial solar nebula. Icarus 45:517-28

Nakagawa Y, Sekiya M, Hayashi C. 1986. Settling and growth of dust particles in a laminar phase of a low-mass solar nebula. Icarus 67:375-90

Nelson RP, Papaloizou JCB, Massét F, Kley W. 2001. The migration and growth of protoplanets in protostellar discs. MNRAS 318:18-36

Nutzman P, Charbonneau D. 2008. Design considerations for a ground-based transit search for habitable planets orbiting M dwarfs. Publ. Astron. Soc. Pac. 120:317-27

O'Brien DP, Morbidelli A, Levison HF. 2006. Terrestrial planet formation with strong dynamical friction. Icarus 184:39-58

Okuzumi S, Tanaka H, Kobayashi H, Wada K. 2012. Rapid coagulation of porous dust aggregates outside the snow line: a pathway to successful icy planetesimal formation. Astrophys. J. 752:106-23 
O'Neill C, Lenardic A. 2007. Geological consequences of super-sized Earths. Geophys. Res. Lett. 34:L19204

Ormel CW, Cuzzi JN. 2007. Closed-form expressions for particle relative velocities induced by turbulence. Astrophys. Astron. 466:413-20

Pan L, Padoan P, Scalo J, Kritsuk AG, Norman ML. 2011. Turbulent clustering of protoplanetary dust and planetesimal formation. Astrophys. J. 740:6

Papaloizou JCB, Terquem C. 2006. Planet formation and migration. Rep. Prog. Phys. 69:119-80

Peale SJ, Lee MH. 2002. A primordial origin of the Laplace relation among the Galilean satellites. Science 298:593-97

Podolak M. 2003. The contribution of small grains to the opacity of protoplanetary atmospheres. Icarus 165:428-37

Pollack JB, Hubickyj O, Bodenheimer P, Lissauer JJ, Podolak M, Greenzweig Y. 1996. Formation of the giant planets by concurrent accretion of solids and gas. Icarus 124:62-85

Queloz D, Bouchy F, Moutou C, Hatzes A, Hébrard G, et al. 2009. The CoRoT-7 planetary system: two orbiting super-Earths. Astron. Astrophys. 506:303-19

Raymond SN, Barnes R, Mandell AM. 2008. Observable consequences of planet formation models in systems with close-in terrestrial planets. MNRAS 384:663-74

Raymond SN, Mandell AM, Sigurdsson S. 2006a. Exotic earths: forming habitable worlds with giant planet migration. Science 313:1413-16

Raymond SN, O'Brien DP, Morbidelli A, Kaib NA. 2009. Building the terrestrial planets: constrained accretion in the inner Solar System. Icarus 203:644-62

Raymond SN, Quinn T, Lunine JI. 2004. Making other earths: dynamical simulations of terrestrial planet formation and water delivery. Icarus 168:1-17

Raymond SN, Quinn T, Lunine JI. 2005a. Terrestrial planet formation in disks with varying surface density profiles. Astrophys. J. 632:670-76

Raymond SN, Quinn T, Lunine JI. 2005b. The formation and habitability of terrestrial planets in the presence of close-in giant planets. Icarus 177:256-63

Raymond SN, Quinn T, Lunine JI. 2006b. High-resolution simulations of the final assembly of Earth-like planets 1: terrestrial accretion and dynamics. Icarus $183: 265-82$

Raymond SN, Quinn T, Lunine JI. 2007. High resolution simulations of the final assembly of Earth-like planets 2: water delivery and planetary habitability. 
Astrobiol. J. 7:66-84

Rivera EJ, Laughlin G, Butler RP, Vogt SS, Haghighipour N, Meschiari S. 2010. TheLick-CarnegieExoplanet Survey: a Uranus-mass fourth planet for GJ 876 in an extrasolar Laplace configuration. Astrophys. J. 719:890-99

Rivera EJ, Lissauer JJ, Butler RP, Marcy GW, Vogt SS, et al. 2005. A 7.5 $M_{\oplus}$ planet orbiting the nearby star, GJ 876. Astrophys. J. 634:625-40

Rogers LA, Bodenheimer P, Lissauer J, Seager S. 2011. Formation and structure of low-density exo-Neptunes. Astrophys. J. 738:59

Rogers LA, Seager S. 2010a. A framework for quantifying the degeneracies of exoplanet interior compositions. Astrophys. J. 712:974-91

Rogers LA, Seager S. 2010b. Three possible origins for the gas layer on GJ 1214b. Astrophys. J. 716:1208-16

Roy AE. 1982. Orbital Motion. Bristol, UK: Adam Hilger

Safronov VS. 1969. Evolution of Protoplanetary Cloud and Formation of the Earth and Planets. Moscow: Nauka

Schlichting E, Warren PH, Yin Q-Z. 2012. The last stages of terrestrial planet formation: dynamical friction and the late veneer. Astrophys. J. 752:8-15

Seager S, Deming D. 2009. On the method to infer an atmosphere on a tidally locked super Earth exoplanet and upper limits to GJ 876d. Astrophys. J. 703:1884-89

Shariff K, Cuzzi JN. 2011. Gravitational instability of solids assisted by gas drag: slowing by turbulent mass diffusivity. Astrophys. J. 738:73

Shi J-M, Chiang E. 2013. From dust to planetesimals: criteria for gravitational instability of small particles in gas. Astrophys. J. 764:20

Smoluchowski MV. 1916. Drei Vortrage uber diffusion, brownsche bewegung und koagulation von Kolloidteilchen. Physik. Zeit. 17:557-85

Sotin C, Schubert G. 2009. Mantle convection and plate tectonics on Earth-like exoplanets. Presented at Am. Geophys. Union Fall Meet., Dec. 14-18, San Francisco (Abstr. P42B-02)

Strom SE, Edwards S, Skrutskie MF. 1993. Evolutionary time scales for circumstellar disks associated with intermediate- and solar-type stars. In Protostars and Planets III, ed. EH Levy, JI Lunine, pp. 837-66. Tucson: Univ. Ariz. Press

Supulver KD, Lin DNC. 2000. Growth and sedimentation of dust grains in the primordial solar nebula. Icarus 146:525-40 
Swift J, Johnson JA, Morton TD, Crepp JR, Montet BT, et al. 2013. Characterizing the cool KOIs. IV.Kepler-32 as a prototype for the formation of compact planetary systems throughout the galaxy. Astrophys. J. 764:105

Tackley PJ, van Heck H. 2009. Mantle convection, stagnant lids and plate tectonics on super-Earths. Geochim. Cosmochim. Acta 73:A1303

Terquem C, Papaloizou JCB. 2007. Migration and the formation of systems of hot super-Earths and Neptunes. Astrophys. J. 654:1110-20

Torres K, Winter OC, Izidoro A, Haghighipour N. 2013. A compound model for the origin of Earths water. Astrophys. J. 767:54

Tsiganis K, Gomes R, Morbidelli A, Levison HF. 2005. Origin of the orbital architecture of the giant planets of the Solar System. Nature 435:459-61

Udry S, Bonfils X, Delfosse X, Forveille T, Mayor M, et al. 2007. The HARPS search for southern extra-solar planets. XI. Super-Earths (5 and $\left.8 M_{\oplus}\right)$ in a 3-planet system. Astron. Astrophys. 469:L43-47

Valencia D, Ikoma M, Guillot T, Nettelmann N. 2010. Composition and fate of short-period super-Earths. The case of CoRoT-7b. Astron. Astrophys. 516:A20

Valencia D, O'Conell RJ. 2009. Convection scaling and subduction on Earth and super-Earths. Earth Planet. Sci. Lett. 286:492-502

Valencia D, O'Connell RJ, Sasselov DD. 2006. Internal structure of massive terrestrial planets. Icarus 181:545-54

Valencia D, O'Connell RJ, Sasselov DD. 2007a. Inevitability of plate tectonics on super-Earths. Astrophys. J. 670:L45-48

Valencia D, Sasselov DD, O'Connell RJ. 2007b. Detailed models of super-Earths: How well can we infer bulk properties? Astrophys. J. 665:1413-20

Valencia D, Sasselov DD, O'Connell RJ. 2007c.Radius and structure models of the first terrestrial super-Earth planets. Astrophys. J. 656:545-51

Vogt SS, Butler RO, Haghighipour N. 2012. GJ 581 update: additional evidence for a super-Earth in the habitable zone. Astron. Nachr. 333:561-75

Vogt SS, Butler RP, Rivera EJ, Haghighipour N, Henry GW, Williamson MH. 2010. The Lick-Carnegie Exoplanet Survey: A 3.1 $M_{\oplus}$ planet in the habitable zone of the nearby M3V star Gliese 581. Astrophys. J. 723:954-65

Völk HJ, Jones FC, Morfill GE, Roeser S. 1980. Collisions between grains in a turbulent gas. Astron. Astrophys. 85:316-25 
Wada K, Tanaka H, Suyama T, Kimura H, Yamamoto T. 2007. Numerical simulation of dust aggregate collisions. I. Compression and disruption of twodimensional aggregates. Astrophys. J. 661:320-33

Walsh KJ, Morbidelli A, Raymond SN, O'Brien DP, Mandell AM. 2011. A low mass for Mars from Jupiters early gas-driven migration. Nature 475:206-9

Weidenschilling SJ. 1977. Aerodynamics of solid bodies in the solar nebula. MNRAS 180:57-70

Weidenschilling SJ. 1980. Growth and sedimentation of dust grains in the primordial solar nebula. Icarus 44:172-89

Weidenschilling SJ. 2010. Particles in the nebular midplane: collective effects and relative velocities. Meteorit. Planet. Sci. 45:276-88

Weidenschilling SJ, Spaute D, Davis DR, Marzari F, Ohtsuki K. 1997. Accretional evolution of a planetesimal swarm. Icarus 128:429-55

Wetherill GW. 1990a. Comparison of analytical and physical modeling of planetesimal accumulation. Icarus 88:336-54

Wetherill GW. 1990b. Formation of earth. Annu. Rev. Earth Planet. Sci. 18:205-56

Wetherill GW. 1994. Provenance of the terrestrial planets. Geochim. Cosmochim. Acta 58:4513-20

Wetherill GW. 1996. The formation and habitability of extra-solar planets. Icarus 119:219-38

Wetherill GW, Stewart GR. 1989. Accumulation of a swarm of small planetesimals. Icarus 77:330-57

Wetherill GW, Stewart GR. 1993. Formation of planetary embryos-effects of fragmentation, low relative velocity, and independent variation of eccentricity and inclination. Icarus 106:190-209

Whipple FL. 1972. On certain aerodynamic processes for asteroid and comets. Proc. Nobel Symp., 21st, Saltsjöbaden, Sweden, Sept. 6-10, 1971, ed. A Elvius, pp. 211-32. New York: Wiley

Wolszczan A, Frail DA. 1992. A planetary system around the millisecond pulsar PSR 1257+12. Nature 355:145-47

Wurm G, Blum J. 1998. Experiments on preplanetary dust aggregation. Icarus $132: 125-36$

Zhou J-L, Aarseth SJ, Lin DNC, Nagasawa M. 2005. Origin and ubiquity of 
short-period Earth-like planets: evidence for the sequential accretion theory of planet formation. Astrophys. J. 631:L85-88

Zsom A, Ormel CW, Güttler C, Blum J, Dullemond CP. 2010. The first phase of protoplanetary dust growth: the bouncing barrier. Astron. Astrophys. 513:A57 
Table 1: Currently known extrasolar planets with masses up to 10 Earth-masses. The quantities $M, P, a$ and $e$ represent the mass (in terms of Earth's mass $M_{\oplus}$ ), orbital period, semimajor axis, and orbital eccentricity of the planet. The mass of the central star is shown by $M_{*}$ and is given in the units of solar-masses $\left(M_{\odot}\right)$.

\begin{tabular}{|c|c|c|c|c|c|c|}
\hline Planet & $\overline{M\left(M_{\oplus}\right)}$ & $P$ (day) & $a(\mathrm{AU})$ & $e$ & Stellar Type & $M_{*}\left(M_{\odot}\right)$ \\
\hline KOI-55 c & 0.6678 & 0.34289 & 0.0076 & - & 0.496 & $\mathrm{sdB}$ \\
\hline Kepler-42 d & 0.954 & 1.856169 & 0.0154 & - & 0.13 & - \\
\hline Kepler-42 c & 1.908 & 0.45328509 & 0.006 & - & 0.13 & - \\
\hline Gl 581 e & 1.9398 & 3.14945 & 0.028 & 0.32 & 0.31 & $\mathrm{M} 2.5 \mathrm{~V}$ \\
\hline Kepler-11 f & 2.301366 & 46.68876 & 0.25 & 0 & 0.95 & G \\
\hline HD $20794 \mathrm{c}$ & 2.4168 & 40.114 & 0.2036 & 0 & 0.7 & G8V \\
\hline HD 20794 b & 2.703 & 18.315 & 0.1207 & 0 & 0.7 & G8V \\
\hline HD 215152 b & 2.7666 & 7.2825 & 0.0652 & 0.34 & - & K0 \\
\hline Kepler-42 b & 2.862 & 1.2137672 & 0.0116 & - & 0.13 & - \\
\hline HD 215152 c & 3.0846 & 10.866 & 0.0852 & 0.38 & - & K0 \\
\hline Kepler-20 e & 3.0846 & 6.098493 & 0.0507 & & 0.912 & G8 \\
\hline $\begin{array}{c}\text { MOA-2007-BLG } \\
-192-\mathrm{L} b\end{array}$ & 3.18 & - & 0.66 & - & 0.06 & M \\
\hline Kepler-32 b & 3.4 & 5.90 & 0.0519 & - & 0.54 & M1V \\
\hline HD 85512 b & 3.498 & 58.43 & 0.26 & 0.11 & 0.69 & K5V \\
\hline HD 39194 b & 3.7206 & 5.6363 & 0.0519 & 0.2 & - & $\mathrm{K} 0 \mathrm{~V}$ \\
\hline Kepler-32 c & 3.8 & 8.75 & 0.067 & - & 0.54 & M1V \\
\hline PSR $1257+12$ d & 3.816 & 98.2114 & 0.46 & 0.025 & - & - \\
\hline PSR $1257+12$ c & 4.134 & 66.5419 & 0.36 & 0.018 & - & - \\
\hline HD 156668 b & 4.1658 & 4.646 & 0.05 & 0 & 0.772 & K3V \\
\hline HD $40307 \mathrm{~b}$ & 4.1976 & 4.3115 & 0.047 & 0 & 0.77 & $\mathrm{~K} 2.5 \mathrm{~V}$ \\
\hline GJ $667 \mathrm{C} \mathrm{c}$ & 4.2612 & 28.13 & 0.1251 & 0.34 & 0.33 & $\mathrm{M} 1.5 \mathrm{~V}$ \\
\hline Kepler-11 b & 4.30254 & 10.30375 & 0.091 & 0 & 0.95 & G \\
\hline KOI-55 b & 4.452 & 0.2401 & 0.006 & - & 0.496 & sdB \\
\hline Kepler-10 b & 4.5474 & 0.837495 & 0.01684 & 0 & 0.895 & $\mathrm{G}$ \\
\hline HD $20794 \mathrm{~d}$ & 4.77 & 90.309 & 0.3499 & 0 & 0.7 & G8V \\
\hline CoRoT-7 b & 4.8018 & 0.853585 & 0.0172 & 0 & 0.93 & K0V \\
\hline 61 Vir b & 5.088 & 4.215 & 0.050201 & 0.12 & 0.95 & G5V \\
\hline HD 39194 d & 5.1516 & 33.941 & 0.172 & 0.2 & - & K0V \\
\hline HD 136352 b & 5.2788 & 11.577 & 0.0933 & 0.18 & - & G4V \\
\hline Gl 581 c & 5.406 & 12.9182 & 0.073 & 0.07 & 0.31 & $\mathrm{M} 2.5 \mathrm{~V}$ \\
\hline
\end{tabular}


Table 2: Continuing from Table 1. Currently known extrasolar planets with masses up to 10 Earth-masses. The quantities $M, P, a$ and $e$ represent the mass (in terms of Earth's mass $M_{\oplus}$ ), orbital period, semimajor axis, and orbital eccentricity of the planet. The mass of the central star is shown by $M_{*}$ and is given in the units of solar-masses $\left(M_{\odot}\right)$.

\begin{tabular}{lllllll}
\hline Planet & $M\left(M_{\oplus}\right)$ & $P($ day $)$ & $a(\mathrm{AU})$ & $e$ & Stellar Type & $M_{*}\left(M_{\odot}\right)$ \\
\hline OGLE-2005-390L b & 5.406 & 3500 & 2.1 & - & 0.22 & $\mathrm{M}$ \\
GJ 667C b & 5.46324 & 7.199 & 0.0504 & 0.09 & 0.33 & $\mathrm{M} 1.5 \mathrm{~V}$ \\
GJ 433 b & 5.7876 & 7.3709 & 0.058 & 0.08 & 0.48 & $\mathrm{M} 1.5$ \\
HD 1461 c & 5.9148 & 13.505 & 0.1117 & 0 & 1.08 & $\mathrm{G} 0 \mathrm{~V}$ \\
HD 39194 c & 5.9466 & 14.025 & 0.0954 & 0.11 & - & $\mathrm{K} 0 \mathrm{~V}$ \\
Gl 581 d & 6.042 & 66.64 & 0.22 & 0.25 & 0.31 & $\mathrm{M} 2.5 \mathrm{~V}$ \\
Kepler-11 d & 6.10242 & 22.68719 & 0.159 & 0 & 0.95 & $\mathrm{G}$ \\
HD 154088 b & 6.1374 & 18.596 & 0.1316 & 0.38 & - & $\mathrm{K} 0 \mathrm{IV}$ \\
GJ 1214 b & 6.36 & 1.58040482 & 0.014 & 0.27 & 0.153 & $\mathrm{M}$ \\
HD 215497 b & 6.36 & 3.93404 & 0.047 & 0.16 & 0.87 & $\mathrm{~K} 3 \mathrm{~V}$ \\
HD 97658 b & 6.36 & 9.4957 & 0.0797 & 0.13 & 0.85 & $\mathrm{~K} 1 \mathrm{~V}$ \\
Gl 876 d & 6.678 & 1.93778 & 0.0208 & 0.21 & 0.334 & $\mathrm{M} 4 \mathrm{~V}$ \\
HD 40307 c & 6.8688 & 9.62 & 0.081 & 0 & 0.77 & $\mathrm{~K} 2.5 \mathrm{~V}$ \\
Kepler-18 b & 6.9006 & 3.504725 & 0.0447 & - & 0.972 & - \\
GJ 3634 b & 6.996 & 2.64561 & 0.0287 & 0.08 & 0.45 & $\mathrm{M} 2.5$ \\
Kepler-9 d & 6.996 & 1.592851 & 0.0273 & - & 1 & - \\
HD 181433 b & 7.5684 & 9.3743 & 0.08 & 0.39 & 0.78 & $\mathrm{~K} 3 \mathrm{IV}$ \\
HD 1461 b & 7.6002 & 5.7727 & 0.063 & 0.14 & 1.08 & $\mathrm{G} 0 \mathrm{~V}$ \\
HD 93385 b & 8.3634 & 13.186 & 0.1116 & 0.15 & - & $\mathrm{G} 2 \mathrm{~V}$ \\
CoRoT-7 c & 8.3952 & 3.698 & 0.046 & 0 & 0.93 & K0V \\
Kepler-11 e & 8.40474 & 31.9959 & 0.194 & 0 & 0.95 & $\mathrm{G}$ \\
GJ 176 b & 8.427 & 8.7836 & 0.066 & 0 & 0.49 & M2.5V \\
55 Cnc e & 8.586 & 0.7365449 & 0.0156 & 0.06 & 0.905 & K0IV-V \\
Kepler-20 b & 8.586 & 3.6961219 & 0.0453 & 0.32 & 0.912 & G8 \\
HD 96700 b & 9.0312 & 8.1256 & 0.0774 & 0.1 & - & G0V \\
HD 40307 d & 9.1584 & 20.46 & 0.134 & 0 & 0.77 & K2.5V \\
HD 7924 b & 9.222 & 5.3978 & 0.057 & 0.17 & 0.832 & KOV \\
HD 134606 b & 9.2856 & 12.083 & 0.102 & 0.15 & - & G6IV \\
HD 136352 d & 9.54 & 106.72 & 0.411 & 0.43 & - & G4V \\
HD 189567 b & 10.0488 & - & 14.275 & 0.11 & 0.23 & G2V \\
HD 93385 c & 10.1124 & - & 46.025 & 0.21 & 0.24 & G2V \\
\hline
\end{tabular}




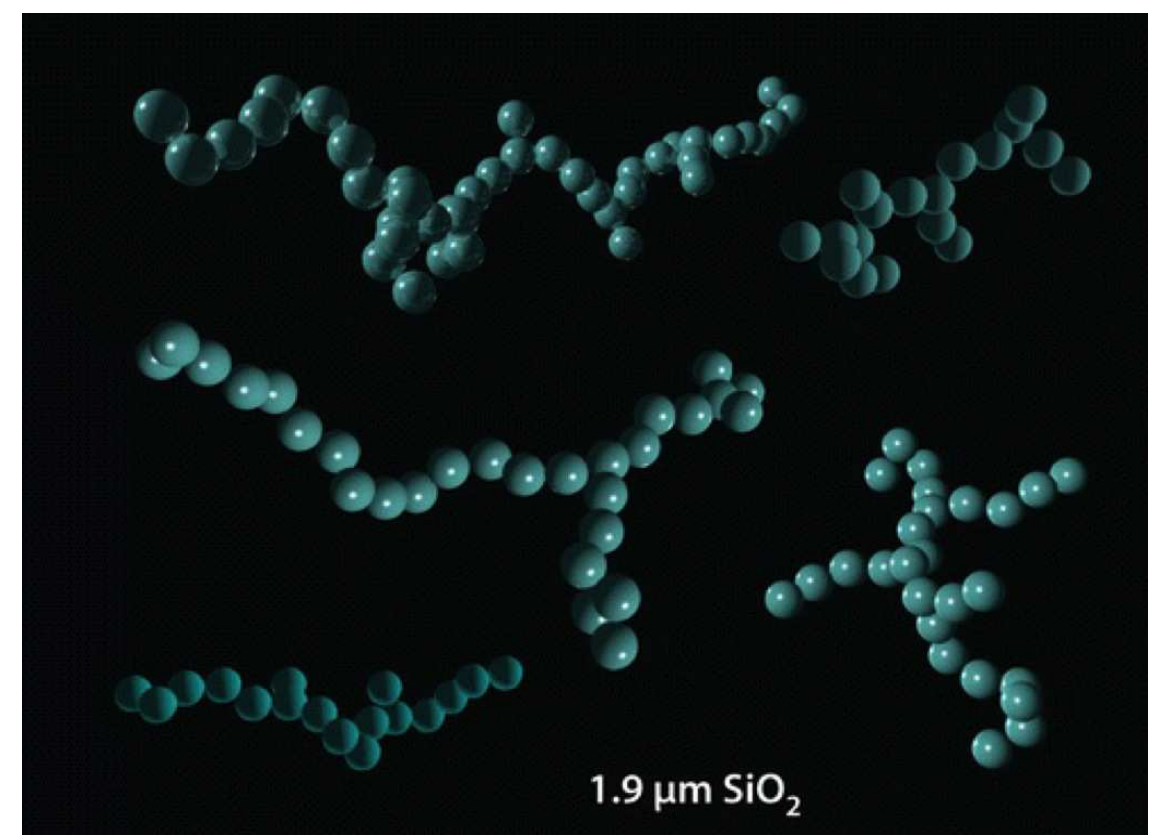

Figure 1: Coagulation of dust particles to fractal aggregates. Figure courtesy of J. Blum. 


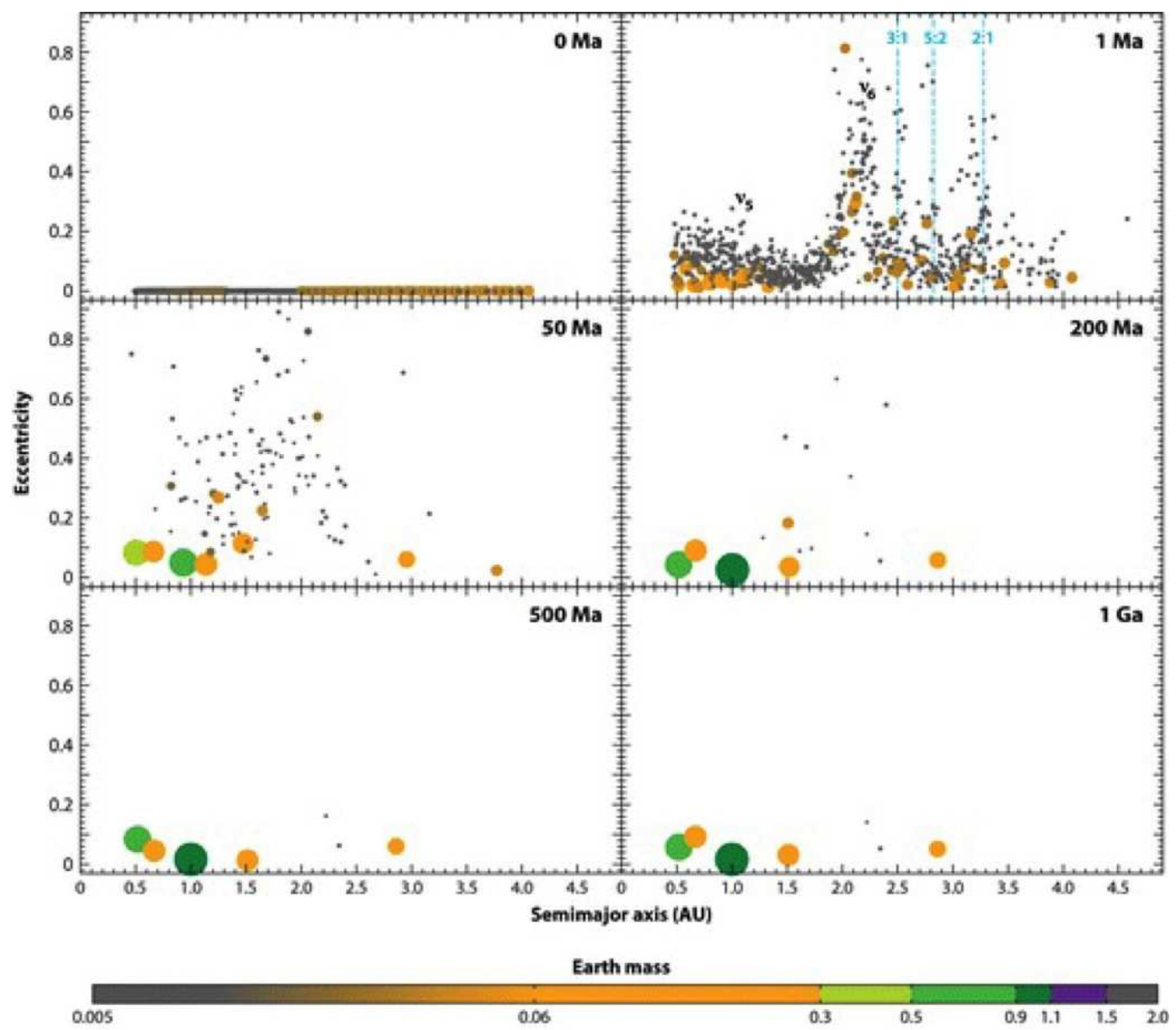

Figure 2: Snapshots of the accretion of planetesimals and planetary embryos to terrestrial-size planets. The disk has a radial surface density profile of -1.5 with its value at $1 \mathrm{AU}$ equal to $8 \mathrm{~g} \mathrm{~cm}^{-3}$. Mean-motion and secular resonances with Jupiter and Saturn are also shown. Figure courtesy of A. Izidoro. 

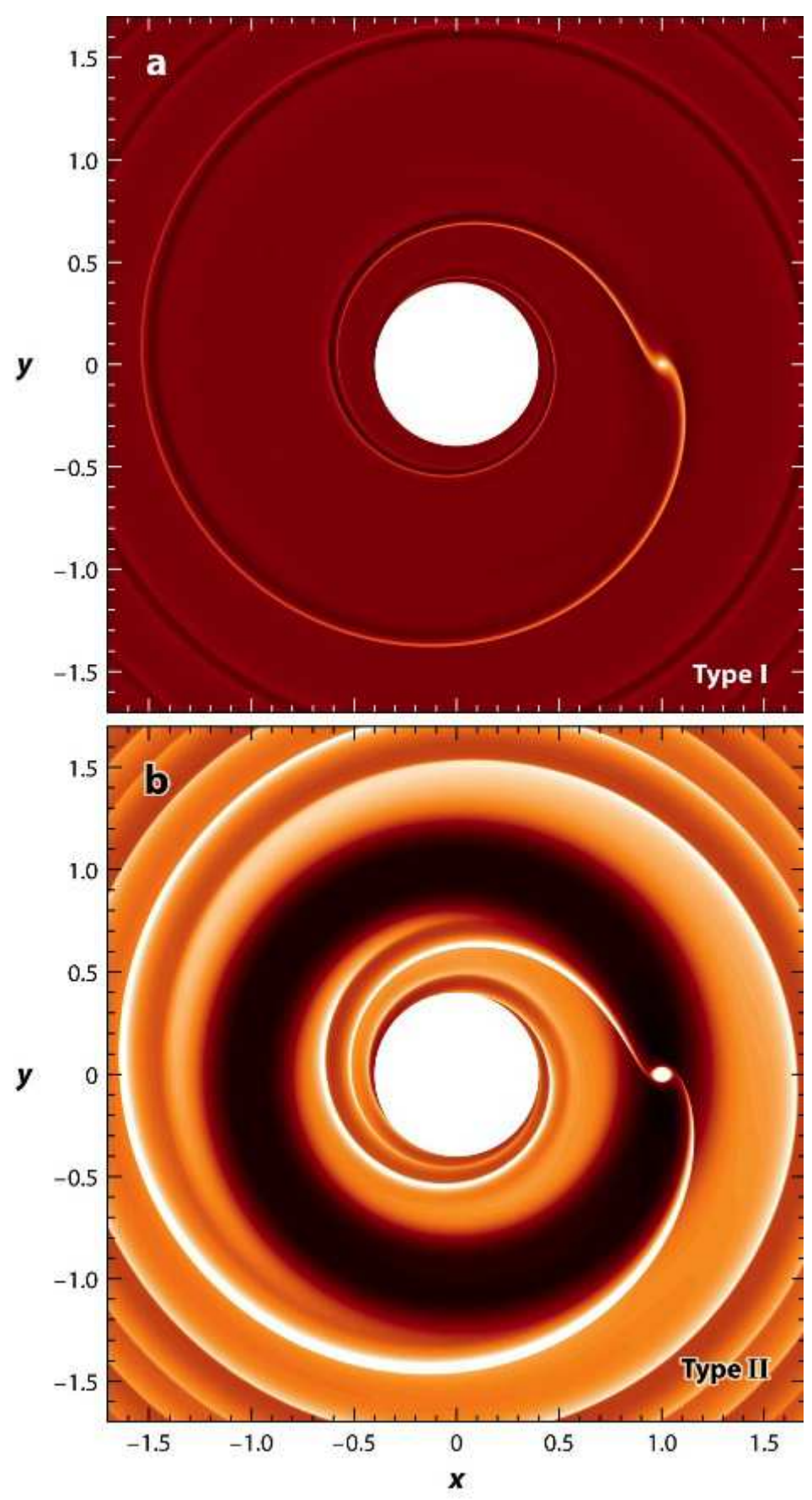

Figure 3: Type I (top) and type II (bottom) planetary migration. Figures courtesy of F. Massét. 


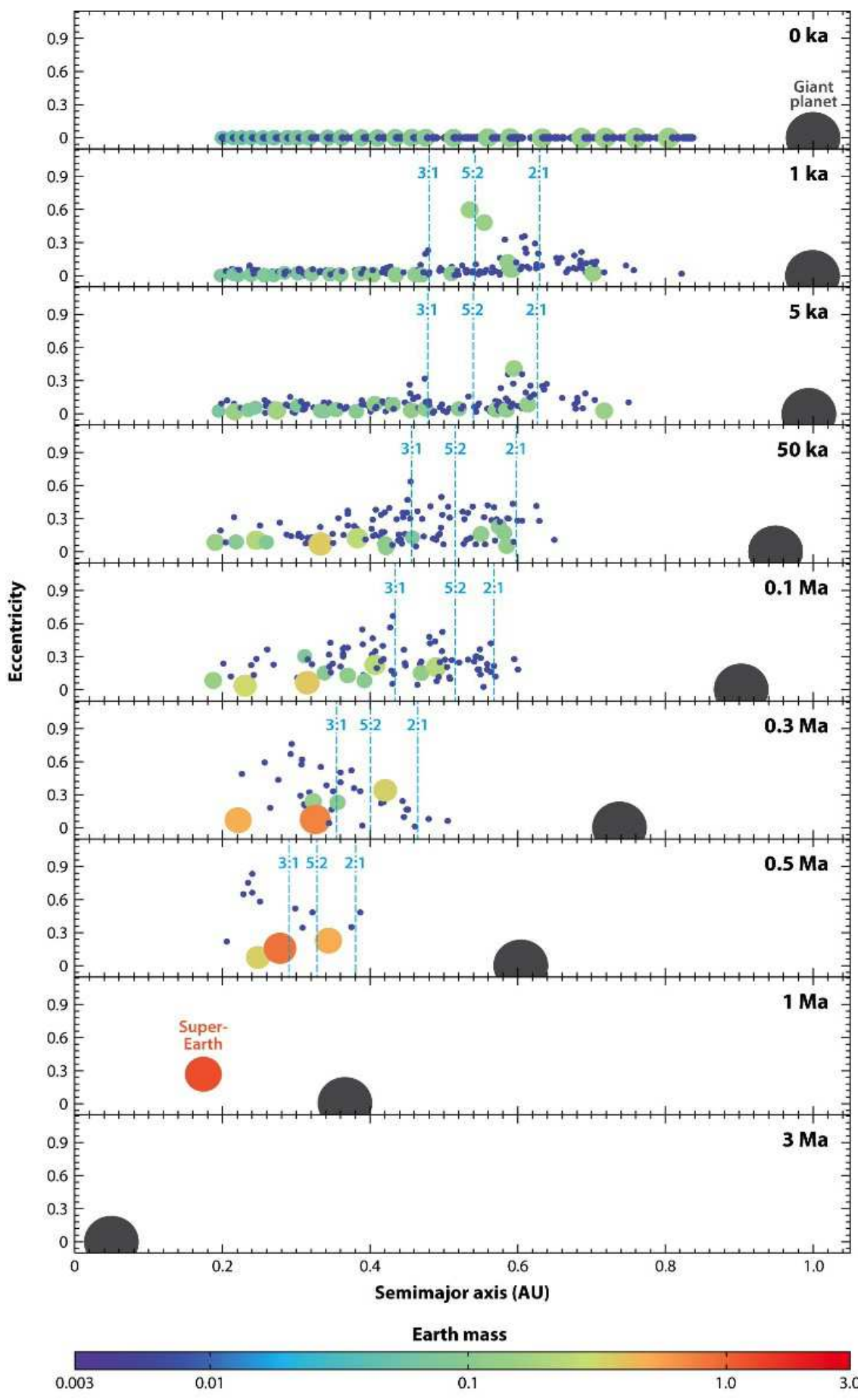

Figure 4: Accretion of protoplanetary bodies during the migration of a giant planet around a $0.3 M_{\odot} \mathrm{M}$ star (Haghighipour \& Rastegar 2011). 


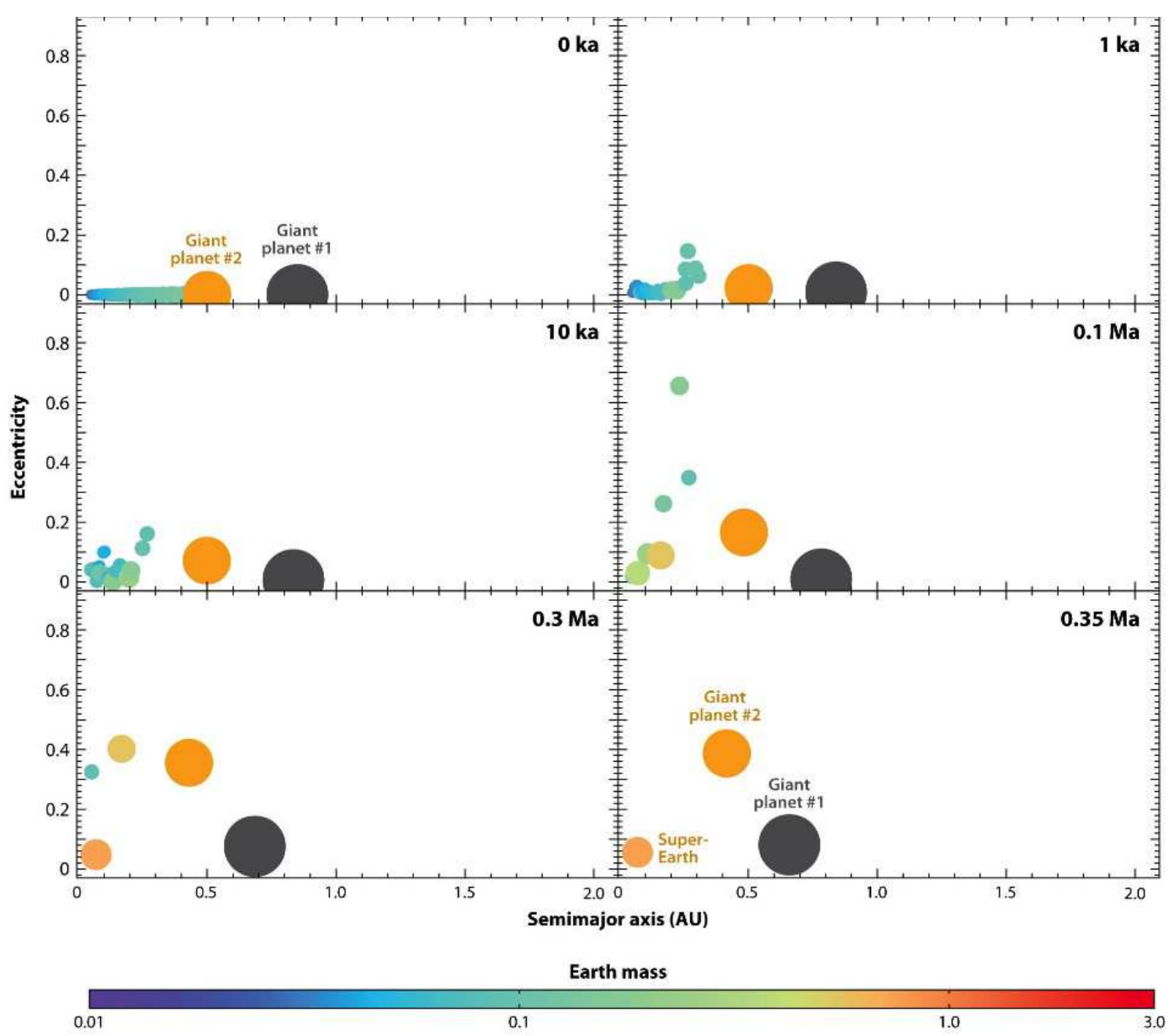

Figure 5: Accretion of protoplanetary bodies during the migration of two giant planets around a $0.3 M_{\odot} \mathrm{M}$ star. As shown here, the system becomes stable with two giant planets in a 1:2 MMR and a super-Earth in a short-period orbit (e.g., GJ 876). 

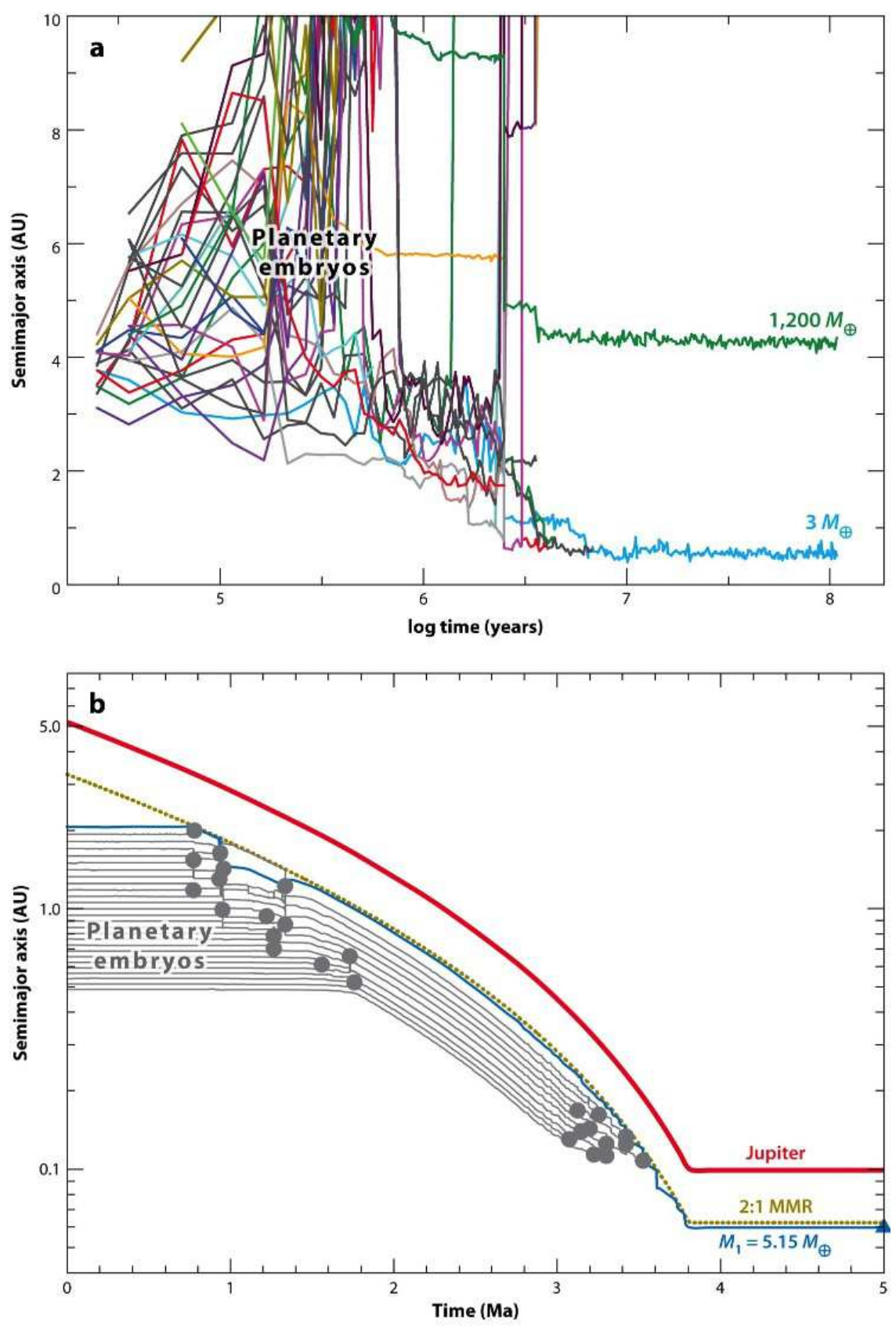

Figure 6: Migration and accretion of planetary embryos and the formation of super-Earths. Top: The formation of an icy $3 M_{\oplus}$ object at 0.5 AU. The superEarth has two giant companions, one at $10 \mathrm{AU}$ (not shown here) and one at $4 \mathrm{AU}$ with a mass of 1,200 $M_{\oplus}$. Figure courtesy of S. Kenyon. Bottom: A combination of the migration and accretion of embryos to super-Earth bodies and their capture in MMR resonances. Figure courtesy of J.-L. Zhou. 


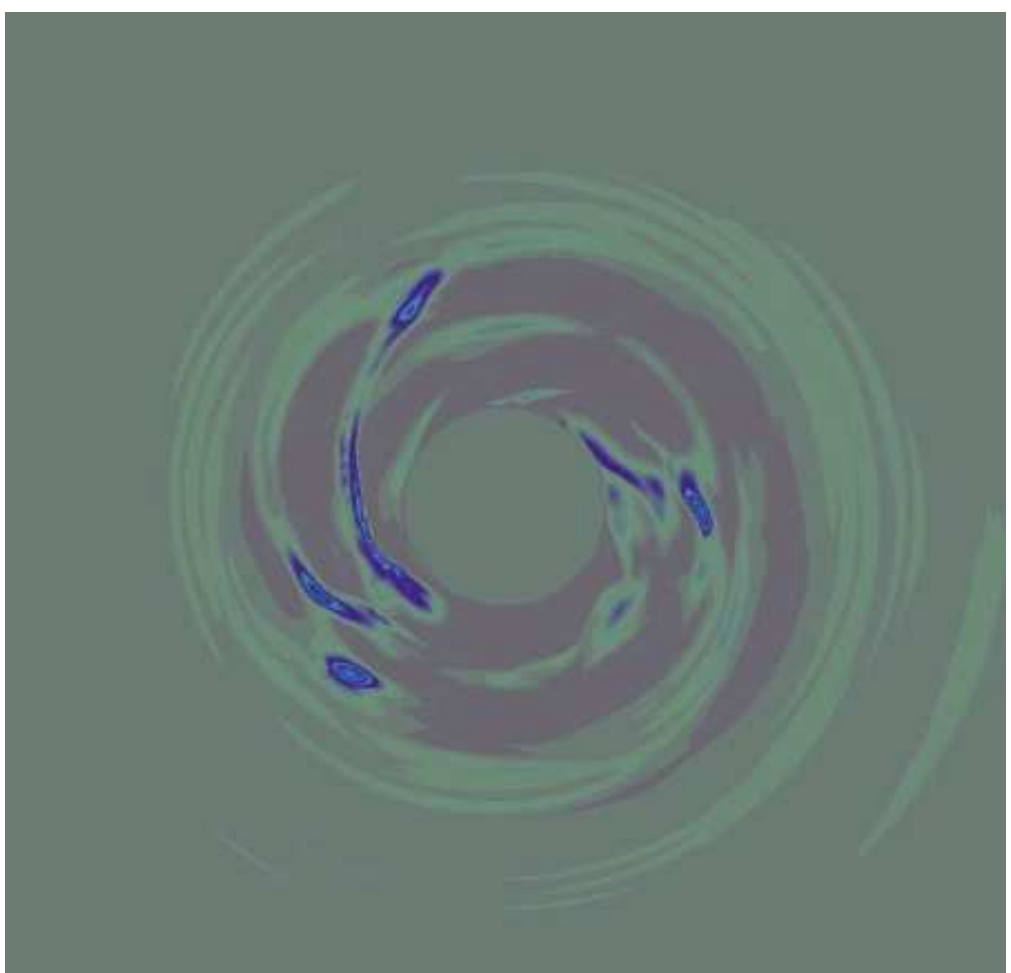

Figure 7: A snapshot of a simulation of the formation of super-Earths around a $0.5 M_{\odot}$ star in the disk-instability model. The four clumps shown in light blue are potential mini-Neptune and super-Earth objects. Figure courtesy of A. Boss. 